\title{
Measurement Techniques for Low Emittance Tuning and Beam Dynamics at CESR
}

\author{
M.G. Billing, J.A. Dobbins, M.J. Forster, D.L. Kreinick, R.E. Meller, D.P. Peterson, G.A. \\ Ramirez, M.C. Rendina, N.T. Rider, D.C. Sagan, J. Shanks, J.P. Sikora, M.G. Stedinger, C.R. \\ Strohman, H.A. Williams, Cornell Laboratory for Accelerator-based ScienceS and Education \\ (CLASSE), Cornell University, 161 Synchrotron Dr., Ithaca, NY, 14850, U.S.A. \\ M.A. Palmer, Collider Accelerator Department, Brookhaven National Laboratory P.O.Box \\ 5000, Upton, NY 11973-5000, U.S.A. \\ R.L. Holtzapple, Physics Department, California Polytechnic State University, San Luis \\ Obispo, CA 93407, U.S.A. \\ J. Flanagan, High Energy Accelerator Research Organization (KEK), 1-1 Oho, Tsukuba, \\ Ibaraki Prefecture 305-0801, Japan
}

\begin{abstract}
After operating as a High Energy Physics electron-positron collider, the Cornell Electron-positron Storage Ring (CESR) has been converted to become a dedicated synchrotron light source for the Cornell High Energy Synchrotron Source (CHESS). Over the course of several years CESR was adapted for accelerator physics research as a test accelerator, capable of studying topics relevant to future damping rings, colliders and light sources. Initially some specific topics were targeted for accelerator physic research with the storage ring in this mode, labeled CesrTA. These topics included 1) tuning techniques to produce low emittance beams, 2) the study of electron cloud (EC) development in a storage ring and 3) intra-beam scattering effects. The complete conversion of CESR to CesrTA occurred over a several year period, described elsewhere[1-3]. A number of specific instruments were developed for CesrTA. Much of the pre-existing instrumentation was modified to accommodate the scope of these studies and these are described in a companion paper[4]. To complete this research, a number of procedures were developed or modified, often requiring coordinated measurements among different instruments[5]. This paper provides an overview of types of measurements employed for the study of beam dynamics during the operation of CesrTA.
\end{abstract}

Keywords: Accelerator Subsystems and Technologies, Beam-line Instrumentation 


\section{Contents}

1 Introduction 2

2 Overview of the Requirements for Beam Instrumentation 2

2.1 Low Emittance Tuning Measurements 2

2.2 Intra-Beam Scattering Measurements 3

2.3 Electron Cloud Dynamics Measurements 3

3 Beam-based Measurement Techniques 4

3.1 Phase and Coupling Correction 4

3.2 Dispersion 8

$3.3 \mathrm{BPM} /$ quad centering $\quad 8$

3.4 BPM Gains 9

3.4.1 Effect from BPM Gain Errors on Vertical Dispersion 9

$\begin{array}{ll}3.4 .2 & \text { BPM Gain Calibration Procedure }\end{array}$

$\begin{array}{lll}3.5 & \text { BPM Tilts } & 12\end{array}$

4 Performance of Low Emittance Instrumentation 12

$\begin{array}{lll}4.1 \text { Orbit } & 12\end{array}$

$\begin{array}{lll}4.2 & \text { Dispersion } & 13\end{array}$

$\begin{array}{lll}4.3 & \text { Phase and Coupling } & 14\end{array}$

4.4 xBSM - Vertical Beam Size 14

4.5 Summary of Low Emittance Measurement Resolution 15

5 Electron Cloud and Intra-beam Scattering Experimental Hardware and Techniques $\mathbf{1 5}$

5.1 Requirements for Electron Cloud Studies 16

$\begin{array}{lll}5.2 & \text { Bunch-by-Bunch Tune Measurements } & 17\end{array}$

5.2.1 Multi-bunch Large Amplitude Excitation $\quad 17$

$\begin{array}{ll}\text { 5.2.2 Single Bunch Small Amplitude Excitation } & 17\end{array}$

5.2.3 Feedback System Response 19

$\begin{array}{lll}\text { 5.2.4 Self-Excitation } & 20\end{array}$

$\begin{array}{lll}5.3 & \text { Instability Measurements } & 21\end{array}$

5.3.1 Bunch-by-Bunch Position Spectra 23

5.3.2 Bunch-by-Bunch Beam Size 26

5.4 Mode Growth Rates 26

5.4.1 Drive-Damp Excitation: Method 26

5.4.2 Drive-Damp Excitation: Examples 28

$\begin{array}{llr}6 & \text { Summary } & 29\end{array}$ 


\section{Introduction}

The CesrTA project was defined initially with a set of specific goals for accelerator physics research and development:

- The investigation of instrumentation and methodology to systematically reduce the vertical emittance of the stored beam.[6, 7]

- The measurement and characterization of the intra-beam scattering effects as they lead to emittance enlargement.[8-10]

- The study of the effects on the stored positron beam due to electron clouds (EC), produced by synchrotron radiation-induced photoelectrons. The goal is to 1) characterize and quantify the production mechanisms, 2) compare these with computer simulations, 3) develop methods to mitigate the EC effects, and 4) study the related beam dynamics and instabilities.[11-15]

To meet these goals a number of instruments in CESR required significant upgrades or complete development of new designs and subsequent implementation. The simultaneous or coordinated data acquisition of the ensemble of instruments, which is required during specific measurement sequences is described below in the following broad categories:

- Beam-Based Measurement Techniques

- Performance of Low Emittance Instrumentation

- Coordinated Dynamics Measurements

The following sections include descriptions of the measurements that are undertaken and some of the analyses that are performed to meet the goals. Note that these sections are not intended to present detailed accelerator physics measurements as this is reserved for papers, which can present the motivation and the experimental details for the different sets of observations.

\section{Overview of the Requirements for Beam Instrumentation}

The particle beam instrumentation developed specifically for CesrTA or adapted from CESR's existing instrumentation has been employed to study low emittance beams, intra-beam scattering and EC beam dynamics. This section provides an overview for some of the fundamental measurements that have been undertaken in each of these studies.

\subsection{Low Emittance Tuning Measurements}

Tuning methods have been developed, which has allowed a significant reduction of the ratio of the vertical emittance with respect to the horizontal emittance. This method is described in detail in these references[6, 7]. After performing beam position monitor (BPM) gain corrections and centering, the procedure to reduce vertical emittance uses the CESR beam position monitor (CBPM) system[16] in a sequence of measuring and correcting 1) the closed orbit, 2) betatron phase and coupling, and 3) vertical dispersion. Using the x-ray beam size monitor (xBSM)[17], the success of this procedure is determined by measuring the vertical beam size at each step of the process[6]. 


\subsection{Intra-Beam Scattering Measurements}

Studies of intra-beam scattering have been undertaken at CesrTA $[9,18-21]$. The operating tunes for the storage ring were measured using either 1) the set of shakers to drive the beam and then observe a stretched BPM signal on a spectrum analyzer or 2) the tune tracker and turn-by-turn CBPM measurements, which are then Fourier transformed. At the selected tunes and after performing the low emittance tuning procedure described in section 2.1, a set of observations were made as a function of the number of particles in a single bunch. The instruments utilized to record these observations are 1) the xBSM to measure the vertical beam size turn-by-turn, 2) the visible-spectrum beam size monitor (vBSM) to measure the horizontal beam size and 3) a streak camera to measure the bunch length via the visible light port. The data is typically recorded every few seconds as the beam current decays.

\subsection{Electron Cloud Dynamics Measurements}

Photo-electrons produced from synchrotron radiation photons impinging on the vacuum chamber wall are accelerated by stored positron bunches. If several bunches are closely spaced to form a train, then the accelerated electrons impact the vacuum chamber, producing secondary electrons, which are again accelerated. If the surface of the wall has a secondary emission yield larger than one, then the number of electrons in the EC multiply. This cloud can react back on the beam affecting its dynamics. At CesrTA dynamics studies of the EC's interaction with the beam fall into three broad classes of measurements, 1) the tune shift due to the EC through a train of bunches, 2) the beam position spectra and vertical beam sizes of bunches within the train and 3) coherent damping of dipole and head-tail transverse modes vs. the position of bunches within a train[22-24].

Tune shift measurements taken for bunches within the train allow the estimation of the EC density in the neighborhood of the bunches' orbit. The vast majority of the measurements of tune shift have been taken in two different configurations. In the first horizontal and vertical pinger magnets[4] are independently excited to deflect all of the bunches, causing them to execute betatron oscillations in the respective transverse plane. CBPM turn-by-turn data, triggered just before the pingers fire, is acquired for all of the bunches within the train. This process may be repeated as the current in the bunches is uniformly increased. The CBPM data are analyzed offline and the two tunes are identified for each bunch. This method is relatively fast to acquire, but conclusions from these measurements must be tempered as later bunches in the train are actually oscillating in response to the excitation from the moving EC, which itself is being driven at frequencies present from preceding bunches. In CesrTA this effect is especially pronounced in the horizontal plane. The second method uses horizontal and vertical stripline kickers to drive the lead bunch in the train and a second bunch, positioned later in the train. Again CBPM data are taken turn-by-turn for all bunches within the train and the data are Fourier transformed to produce the betatron spectra of the bunches. This method allows an accurate measurement of the tune shift of the later bunch with respect to the lead bunch without driving the EC into an oscillation prior to the arrival of the later bunch. Unlike the first method, this requires taking many sets of data to span the length of the train, but it more accurately reflects the tune shift associated with a "static" EC[25].

Characterizing the instabilities of the bunches within a train due to the $\mathrm{EC}$ is the motivation for the second class of measurements. A train of bunches is filled to a uniform current per bunch, 
at which unstable motion has been detected. Then the gated signal from a single BPM button is detected for a single bunch as is described below. After processing by a spectrum analyzer, the spectrum is recorded bunch-by-bunch. While this process continues to step through the bunches in the train, the xBSM is triggered to measure the beam size of every bunch turn-by-turn. By suitable choices for the settings of the beam stabilizing feedback and the chromatic damping, spectral lines at the head-tail frequencies may be observed. From this data the frequency and equilibrium amplitude of the self-excited bunches and the growth of their vertical beam sizes along the train may be determined[25-30].

The third class of measurements has the same hardware configuration as the preceding class of spectrum measurements. It has been developed to study the damping of coherent betatron motion of the bunches within train to understand any mechanisms, by which the EC cause the motion to damp or de-cohere. This is accomplished by using a stripline kicker, driving one of the transverse dipole or head-tail modes, to excite the motion of a single bunch for a short length of time (e.g. $1 \mathrm{msec}$ ) and then observing the damping of the oscillation using a spectrum analyzer as a tuned receiver at the exciting betatron tune frequency. This measurement provides information about the imaginary part of the tune shift due to the EC[25]. Another variation of drive-damp measurements excites one or both dipole transverse modes of one bunch within a train for a short duration while recording the turn-by-turn positions of all bunches using the CBPM system. Although this second method is only sensitive to the dipole motion of the bunches, it allows the observation of how the motion of one bunch excites subsequent bunches via coupling through the EC.

\section{Beam-based Measurement Techniques}

The low emittance tuning procedure relies on beam based measurements both to calibrate the beam position monitors and to determine sources of emittance dilution. The tuning procedure typically begins with a measurement of the closed orbit. As described in [16] the beam position monitors have a single shot resolution of about $10 \mu \mathrm{m}$. After correcting closed orbit errors, the betatron phase and coupling are measured and corrected as described below. Subsequently the same measurements and corrections are performed for the vertical dispersion. Techniques for determining the linear lattice functions are described later in this section.

Systematic effects limit the accuracy of our measurements of closed orbit, vertical dispersion and, thus, transverse coupling. The most significant systematic error in the measurement of the closed orbit is a result of the uncertainty of the offset of the BPM with respect to the center of the adjacent quadrupole. Beam based BPM/quad centering is discussed below. The accuracy of the measurement of vertical dispersion is limited by variations in the gains of the BPM button electrodes and related electronics and the physical tilt of the beam position monitors. We attempt to resolve these systematics with beam based measurement techniques.

\subsection{Phase and Coupling Correction}

Betatron amplitude, phase and transverse coupling are measured simultaneously. A circulating bunch is driven resonantly with magnetic shakers or stripline kickers by means of tune trackers at the transverse normal mode tunes. Details of this method are described in [31,32], and are briefly summarized here. 


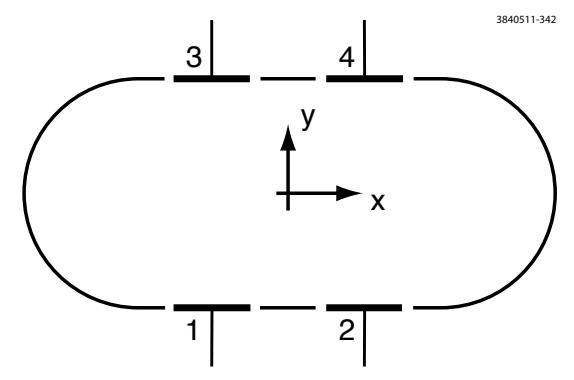

Figure 1. Button layout at a beam position detector

At each of the four button electrodes (see Figure 1) of each beam position monitor, the intensity of the oscillation signal is measured turn-by-turn. Typically $N=40,000$ turns of data are recorded in the CBPM module. For the motion at the "horizontal" frequency the amplitude and phase of the motion at each button is extracted as follows

$$
\begin{aligned}
& A_{j, s i n, h}=\frac{2}{N} \sum_{i=1}^{N} \sin \left(\theta_{t, h}(i)\right) a_{j}(i) \\
& A_{j, \cos , h}=\frac{2}{N} \sum_{i=1}^{N} \cos \left(\theta_{t, h}(i)\right) a_{j}(i)
\end{aligned}
$$

where $A_{j, s i n, h}$ and $A_{j, \cos , h}$ are the "in-phase" and "out-of-phase" components of the horizontal beam motion at button $j=1, \ldots, 4$. In the above equation $\theta_{t, h}(i)$ is the phase of the reference signal from the horizontal tune tracker at turn $i$, which is encoded by the timing system onto the turn-byturn CBPM trigger, and $a_{j}(i)$ is the signal on button $j$ at turn $i$. There is a similar equation for the vertical motion. In order to speed up the data taking process, horizontal and vertical measurements are done simultaneously. The above analysis is performed within the digital signal processor (DSP) of each CBPM module and depends upon the tunes being well enough separated from any low-order resonance so that the "cross-talk" between the horizontal and vertical mode sums in Eqs. 3.1 is negligible. In practice it is always easy to satisfy this condition.

After the button amplitudes have been measured and summed, the in-phase amplitudes in the $x$ and $y$ planes from the horizontal excitation are given by

$$
\begin{aligned}
& A_{x, \sin , h}=C_{x} \frac{A_{4, \sin , h}+A_{2, \sin , h}-A_{3, \sin , h}-A_{1, \sin , h}}{A_{4, \sin , h}+A_{3, \sin , h}+A_{2, \sin , h}+A_{1, \sin , h}} \\
& A_{y, \sin , h}=C_{y} \frac{A_{4, \sin , h}+A_{3, \sin , h}-A_{2, \sin , h}-A_{1, \sin , h}}{A_{4, \sin , h}+A_{3, \sin , h}+A_{2, \sin , h}+A_{1, \sin , h}}
\end{aligned}
$$

Similar equations describe the out-of-phase component at the horizontal tune and then a corresponding set gives the in-phase and out-of-phase response to vertical shaking. $C_{x}$ and $C_{y}$ are spatial constants dependent upon the geometry of the BPM. In the equations above the dependence of position on button amplitude is illustrated assuming linear dependence of the BPM response. In practice we include nonlinear dependence utilizing a nonlinear model of the BPM pickup response[33]. 
The next step is to turn the sine and cosine components into phase and amplitude

$$
\begin{aligned}
& A_{x, h}=\sqrt{\left(A_{x, \sin , h}\right)^{2}+\left(A_{x, \cos , h}\right)^{2}} \\
& \phi_{x, h}=\tan ^{-1} \frac{A_{x, \sin , h}}{A_{x, \cos , h}}
\end{aligned}
$$

and similarly for vertical shaking. In general, the horizontal mode motion of the beam can be written in the form [34]

$$
\begin{aligned}
& x=\gamma_{c} a_{x} \sqrt{\beta_{h}} \cos \left(\psi_{h, i}\right) \\
& y=-a_{x} \sqrt{\beta_{v}}\left[\bar{C}_{22} \cos \left(\psi_{h, i}\right)+\bar{C}_{12} \sin \left(\psi_{h, i}\right)\right]
\end{aligned}
$$

where $\beta_{h}$ and $\beta_{v}$ are the beta functions at the BPM, $\bar{C}_{i j}$ are components of the coupling matrix, and

$$
\psi_{h, i}=i \mu_{h}+\phi_{h, \beta}+\phi_{h, 0}
$$

with $i$ being the turn number, $\mu_{h}$ the horizontal tune, $\phi_{h, \beta}$ the horizontal betatron phase at the BPM, and $\phi_{0}$ an overall phase offset independent of the BPM. In the above equation $\gamma_{c}$ is a parameter dependent upon the coupling. If the local horizontal-to-vertical coupling is small, $\gamma_{c}$ is very nearly unity and can be approximated as such.

Comparison of Eq. 3.4 with the measured beam amplitude components gives

$$
\begin{aligned}
\beta_{h} & =k_{h, \beta} A_{x, h}^{2} \\
\phi_{h, \beta} & =\phi_{x, h}+d \phi_{h} \\
\bar{C}_{12} & =\sqrt{\frac{\beta_{h}}{\beta_{v}}} \frac{A_{y, h}}{A_{x, h}} \sin \left(\phi_{y, h}-\phi_{x, h}\right) \\
\bar{C}_{22} & =-\sqrt{\frac{\beta_{h}}{\beta_{v}}} \frac{A_{y, h}}{A_{x, h}} \cos \left(\phi_{y, h}-\phi_{x, h}\right)
\end{aligned}
$$

where $k_{h, \beta}$ and $d \phi_{h}$ are overall constants dependent upon the amplitude and phase of the shaker drive. A similar analysis is carried out for the vertical shaking data. Here the $x$ and $y$ motions are

$$
\begin{aligned}
& x=a_{y} \sqrt{\beta_{h}}\left[\bar{C}_{11} \cos \left(\psi_{v, i}\right)-\bar{C}_{12} \sin \left(\psi_{v, i}\right)\right] \\
& y=\gamma_{c} a_{y} \sqrt{\beta_{v}} \cos \left(\psi_{v, i}\right)
\end{aligned}
$$

where $\psi_{v, i}$ is analogous to $\psi_{h, i}$ in Eq. 3.5

$$
\psi_{v, i}=i \mu_{v}+\phi_{v, \beta}+\phi_{v, 0}
$$

Comparison of this with the measured beam amplitude components gives

$$
\begin{aligned}
\beta_{v} & =k_{v, \beta} A_{x, v}^{2} \\
\phi_{v, \beta} & =\phi_{x, v}+d \phi_{v} \\
\bar{C}_{12} & =\sqrt{\frac{\beta_{v}}{\beta_{h}}} \frac{A_{x, v}}{A_{y, v}} \sin \left(\phi_{x, v}-\phi_{y, v}\right) \\
\bar{C}_{11} & =-\sqrt{\frac{\beta_{v}}{\beta_{h}}} \frac{A_{x, v}}{A_{y, v}} \cos \left(\phi_{x, v}-\phi_{y, v}\right)
\end{aligned}
$$


A) Before Correction

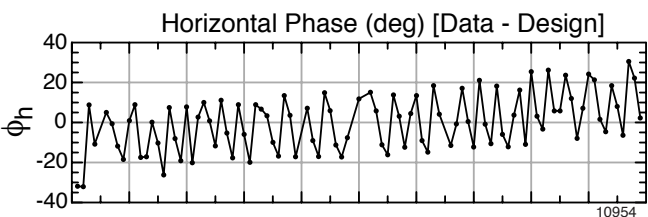

Vertical Phase (deg) [Data - Design]
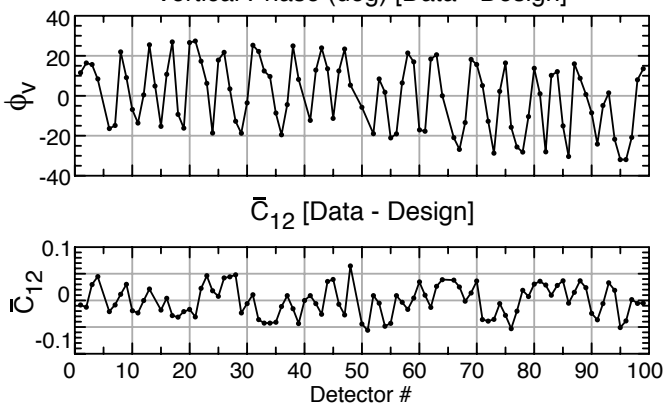

B) After Correction

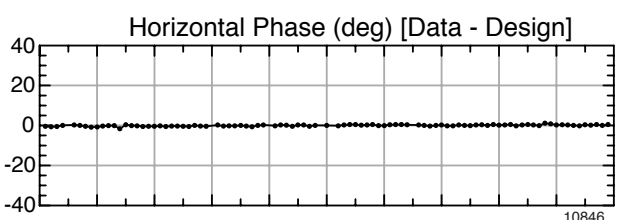

Vertical Phase (deg) [Data - Design]

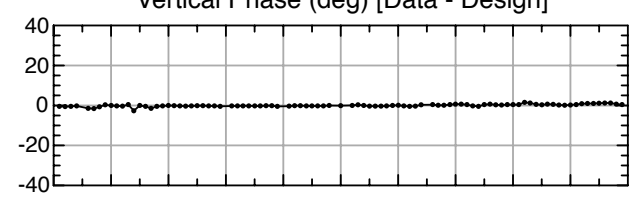

$\overline{\mathrm{C}}_{12}$ [Data - Design]

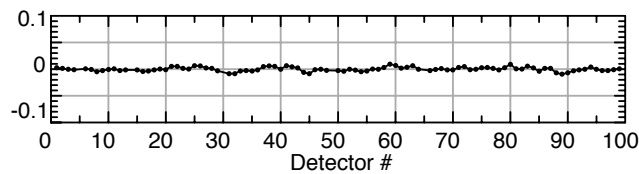

Figure 2. Example betatron phase and coupling correction. A) Measured betatron phases and $\bar{C}_{12}$ coupling matrix element (as computed by equation 3.6) before a correction. Plotted are the difference between the measured values and the design values as computed from the theoretical design lattice. B) Measured values after correction. A perfect correction would result in the measured values being equal to the design ones.

The data is collected and analyzed for all 100 BPMs in less than 10 seconds.

Once a measurement is completed, the independently-controlled strengths of the quadrupoles and skew quadrupoles can be adjusted to correct the Twiss parameters and coupling. This is done using a software model of the CESR lattice. The correction procedure occurs in two steps. The first step is to vary the quadrupoles and skew quadrupoles in the model until the betatron phases and the $\bar{C}_{12}$ as computed from the model most closely match the measured phases and measured $\bar{C}_{12}$. The measured beta is generally ignored since the betatron phase gives a more accurate signal. This is true since the betatron phase is insensitive to variations in the gains of the button signals. Similarly, the $\bar{C}_{11}$ and $\bar{C}_{22}$ data is typically ignored since the $\bar{C}_{12}$ data is generally more accurate. This is due to the insensitivity of the $\bar{C}_{12}$ data to button gain errors and rotations of the BPM in the $x-y$ plane.

An example correction is shown in Figure 2. Figure 2A shows the difference between measured and design betatron phase and coupling before correction. Since the betatron phase can always be offset by an arbitrary additive constant, the phase constants $d \phi_{h}$ and $d \phi_{v}$ are taken to be such that the average measured phase is equal to the average phase as calculated from the design lattice. Figure 2B shows the measured phase and coupling after correction. The deviation between actual and design values is now quite small. The vertical emittance ratio $\epsilon_{v} / \epsilon_{h}$ due to coupling effects is roughly $\sigma_{\bar{C}_{12}}^{2}$, the square of the root mean squared (RMS) standard deviation of $\bar{C}_{12}$. After correction, $\sigma_{\bar{C}_{12}}$ is 0.004 so $\epsilon_{v} / \epsilon_{h}$ due to coupling is of order $2 \times 10^{-5}$, which is very small. The RMS betatron phase deviation after correction is about 0.5 degrees, which translates to an RMS deviation of the actual $\beta$ relative to the design $\beta$ of less than $1 \%$. 


\subsection{Dispersion}

Dispersion is measured via the change in the closed orbit for a given change in beam energy. The beam energy is related to the RF frequency via the momentum compaction, typically $\alpha_{p}=6.8 \times 10^{-3}$ for low emittance CesrTA optics. RF cavity bandwidth and energy aperture limit the frequency change to $\pm 4 \mathrm{kHz}$ of the $500 \mathrm{MHz}$ accelerator cavity frequency and energy offset to about $0.24 \%$. Sensitivity to a vertical dispersion of $1 \mathrm{~cm}$, requires that we measure an orbit difference of $24 \mu \mathrm{m}$. The CesrTA beam position monitors nominally have turn-by-turn reproducibility of order $10 \mu \mathrm{m}$ and the orbit measurement is based on an average of several thousand turns.

\subsection{BPM/quad centering}

To reach the lowest vertical emittance it is necessary for the vertical closed orbit to be as close to the centers of the quadrupoles as possible to minimize vertical kicks, which would contribute to the vertical dispersion. Correction of the closed orbit can be compromised by offsets between the magnetic center of a quadrupole and the electrical center of a nearby BPM.

The standard beam based technique to determine these offsets involves the measurement of orbit changes as the quadrupole's strength $k$ is varied[35]. One common method for determining the quadrupole center involves taking measurements at various beam positions and then interpolating the results to find the quadrupole center, which is the position where the beam orbit does not change with variation of the quadrupole strength. This method can be slow due to the number of measurements needed. For large rings, this technique is not practical.

The measurement method developed at Cornell uses a variation of this procedure:

1. Betatron phases and orbits are measured at two different strength settings for a given quadrupole at some location $s_{q}$. To differentiate the two measurements, one measurement is called the "base" measurement and is denoted with a subscript " 0 " and the other is called the "non-base" measurement.

2. The change in vertical orbit $d y \equiv y-y_{0}$ at the location s, due to the variation in quadrupole strength, is given by

$$
d y(s)=d y^{\prime} \frac{\sqrt{\beta_{y}(s) \beta_{y}\left(s_{q}\right)}}{2 \sin \pi v_{y}} \cos \left(\left|\phi_{y}(s)-\phi_{y}\left(s_{q}\right)\right|-\pi v_{y}\right)
$$

where $d y^{\prime}$ is the kick due to the change in quadrupole strength, and the Twiss parameters $\beta, \phi$ and $v$ are evaluated in the non-base configuration. From the phase data, the Twiss parameters can be calculated. Using the calculated Twiss parameters, a fit to the orbit difference data gives a measurement of $d y^{\prime}$.

3. From a fit of the betatron phase data, the change in quadrupole strength $d k$ and the beta at the quadrupole can be calculated. The change in orbit $d y$ can be written in the form

$$
d y(s)=\left[y_{q c}-y_{0}\left(s_{q}\right)\right] d k L \frac{\sqrt{\beta_{y}(s) \beta_{y}\left(s_{q}\right)}}{2 \sin \pi v_{y}} \cos \left(\left|\phi_{y}(s)-\phi_{y}\left(s_{q}\right)\right|-\pi v_{y}\right)
$$


where $y_{q c}$ is the quadrupole center, and $L$ is the quadrupole length. Comparing the above two equations gives

$$
y_{q c}=\frac{d y^{\prime}}{L d k}+y_{0}\left(s_{q}\right)
$$

A similar analysis holds for the horizontal plane.

4. The accuracy of the calculation depends upon the displacement of the beam from the quadrupole center $d y_{\text {beam }}$. This separation is given by

$$
d y_{\text {beam }}=y_{q c}-y_{0}\left(s_{q}\right)=\frac{d y^{\prime}}{L d k}
$$

The closer the beam is to the quadrupole center, the more accurate the calculation of $y_{q c}$. If the beam is too far off from the center, an orbit bump is used to steer the beam towards the center and the measurement cycle is repeated until $\left|d y_{\text {beam }}\right|$ is within a set tolerance. Typically this is $300 \mu \mathrm{m}$.

The important innovation here is that the analysis incorporates the phase measurement which leads to a more accurate determination of the Twiss parameters and $d k$. This leads to a more accurate determination of $y_{q c}$. The result is that the tolerance on $d y_{\text {beam }}$ can be increased, which leads to a reduced number of measurement cycles and hence a shorter measurement time. The entire procedure in CESR — calibrating all 100 or so BPMs — has been automated and takes somewhat less than two hours.

\subsection{BPM Gains}

The principal systematics limiting the accuracy of our measurement of vertical dispersion are variation of BPM button gains and BPM tilts. There are several effects that will contribute to gain errors, beginning with the button itself and extending through the cabling and electronics. Small variations in how the button is seated in the BPM block will affect the response to the beam signal, as will cable and connector dependent attenuation. Finally, the four BPM button electrodes are connected to dedicated front end amplifiers, each of which can respond with slightly different gains.

\subsubsection{Effect from BPM Gain Errors on Vertical Dispersion}

It is easy to see that the measurement of vertical dispersion is especially sensitive to gain errors. Suppose, for example, at a particular detector there is finite horizontal, but identically zero vertical dispersion. If there is any variation in button gains, a component of horizontal dispersion will appear as vertical dispersion in the measurement. An estimate of the sensitivity of the measurement of vertical dispersion can be made from gain errors with this simple example. Consider a BPM where there is finite horizontal dispersion $\eta_{x}$ and zero vertical dispersion, and the on-energy orbit is at precisely zero in both horizontal and vertical. The position for an energy offset, $+\Delta E / 2$, is given by

$$
x^{+}=x_{0}\left(\frac{B_{4}^{+}-B_{3}^{+}+B_{2}^{+}-B_{1}^{+}}{\sum B_{i}}\right)
$$


where $x_{0}=19.6 \mathrm{~mm}$ is the typical BPM horizontal scale factor and the labels are defined in Figure 1. We assumed that there is zero vertical orbit offset, and since there is zero vertical dispersion and no change in vertical position with the energy, it must be that $B_{4}^{+}=B_{2}^{+}$and $B_{3}^{+}=B_{1}^{+}$so that

$$
x^{+}=2 x_{0}\left(\frac{B_{4}^{+}-B_{3}^{+}}{\sum B_{i}}\right)
$$

Then with energy offset $-\Delta E / 2$,

$$
x^{-}=x_{0}\left(\frac{B_{4}^{-}-B_{3}^{-}+B_{2}^{-}-B_{1}^{-}}{\sum B_{i}}\right)
$$

By symmetry, $B_{3}^{-}=B_{4}^{+}$and $B_{1}^{-}=B_{2}^{+}$. Therefore

$$
\Delta x=x^{+}-x^{-}=4 x_{0}\left(\frac{B_{4}^{+}-B_{3}^{+}}{\sum B_{i}}\right)
$$

and $\eta_{x}=\Delta x / \Delta E$. Now suppose that there is a fractional gain error $f$ for $B_{3}$ so that $B_{3} \rightarrow(1+f) B_{3}$. Then we will measure

$$
y^{+}=y_{0}\left(\frac{f B_{3}^{+}}{\sum B_{i}}\right), \quad y^{-}=y_{0}\left(\frac{f B_{3}^{-}}{\sum B_{i}}\right)=y_{0}\left(\frac{f B_{4}^{+}}{\sum B_{i}}\right)
$$

since $B_{4}^{+}=B_{3}^{-}$. Finally,

$$
\Delta y=y_{0}\left(\frac{f\left(B_{3}^{+}-B_{4}^{+}\right)}{\sum B_{i}}\right)
$$

( $y_{0}=26 \mathrm{~mm}$ for a typical CESR BPM.) Combining the equations 3.18 and 3.20 we find that

$$
\Delta y=\frac{f}{4} \Delta x \frac{y_{0}}{x_{0}} \rightarrow \Delta \eta_{y} \sim 0.34 f \eta_{x}
$$

The peak horizontal dispersion in the CesrTA lattice is about $2 \mathrm{~m}$. Therefore a $1 \%$ gain error on a single button will introduce an error in the measurement of the vertical dispersion of $\Delta \eta_{y} \sim 6.8 \mathrm{~mm}$.

\subsubsection{BPM Gain Calibration Procedure}

To calibrate the gains, we take advantage of the fact that a position measurement in a 4 button detector is over constrained. Indeed, the signals on any three of the four buttons locates the beam in the detector. Expanding the button signals to second order in the beam's displacements, $\mathrm{x}$ and $\mathrm{y}$, we find that the four button signals are related as follows[36]

$$
B_{1}^{i}-B_{2}^{i}-B_{3}^{i}+B_{4}^{i}=\frac{c}{I}\left(B_{1}^{i}-B_{2}^{i}+B_{3}^{i}-B_{4}^{i}\right)\left(B_{1}^{i}+B_{2}^{i}-B_{3}^{i}-B_{4}^{i}\right)
$$


where $B_{j}^{i}$ is the intensity on the $j^{\text {th }}$ button on the $i^{\text {th }}$ measurement. $c$ is a constant determined by the BPM geometry and $I$ the bunch current. If we suppose that there is a variable gain associated with each button then we can write

$$
\begin{aligned}
g_{1} B_{1}^{i}-g_{2} B_{2}^{i}-g_{3} B_{3}^{i}+g_{4} B_{4}^{i} & = \\
& \frac{c}{I}\left(g_{1} B_{1}^{i}-g_{2} B_{2}^{i}+g_{3} B_{3}^{i}-g_{4} B_{4}^{i}\right)\left(g_{1} B_{1}^{i}+g_{2} B_{2}^{i}-g_{3} B_{3}^{i}-g_{4} B_{4}^{i}\right)
\end{aligned}
$$

The next step is to measure response to the beam over the entire active area of the detector so that we have a set $B_{j}^{i}$, for $i=1, \ldots, N$ where $N$ is a large number (typically 1024 turns). Then we fit the $g_{j}$ to minimize

$$
\begin{aligned}
\chi^{2}=\sum_{i=1}^{N}\left[g_{1} B_{1}^{i}-g_{2} B_{2}^{i}-g_{3} B_{3}^{i}+g_{4} B_{4}^{i}-\frac{c}{I}\left(g_{1} B_{1}^{i}-g_{2} B_{2}^{i}+g_{3} B_{3}^{i}-g_{4} B_{4}^{i}\right)\right. \\
\\
\left.\left(g_{1} B_{1}^{i}+g_{2} B_{2}^{i}-g_{3} B_{3}^{i}-g_{4} B_{4}^{i}\right)\right]^{2}
\end{aligned}
$$

In order to sample the active region of all of the BPMs, we resonantly drive the beam simultaneously at the horizontal and vertical tunes, and collect turn-by-turn data. Because the measurement and fitting can be completed in just a few minutes, it is straightforward to maintain up-to-date gain calibrations. An example of fitted gains for all 100 beam position monitors is shown in Figure 3.

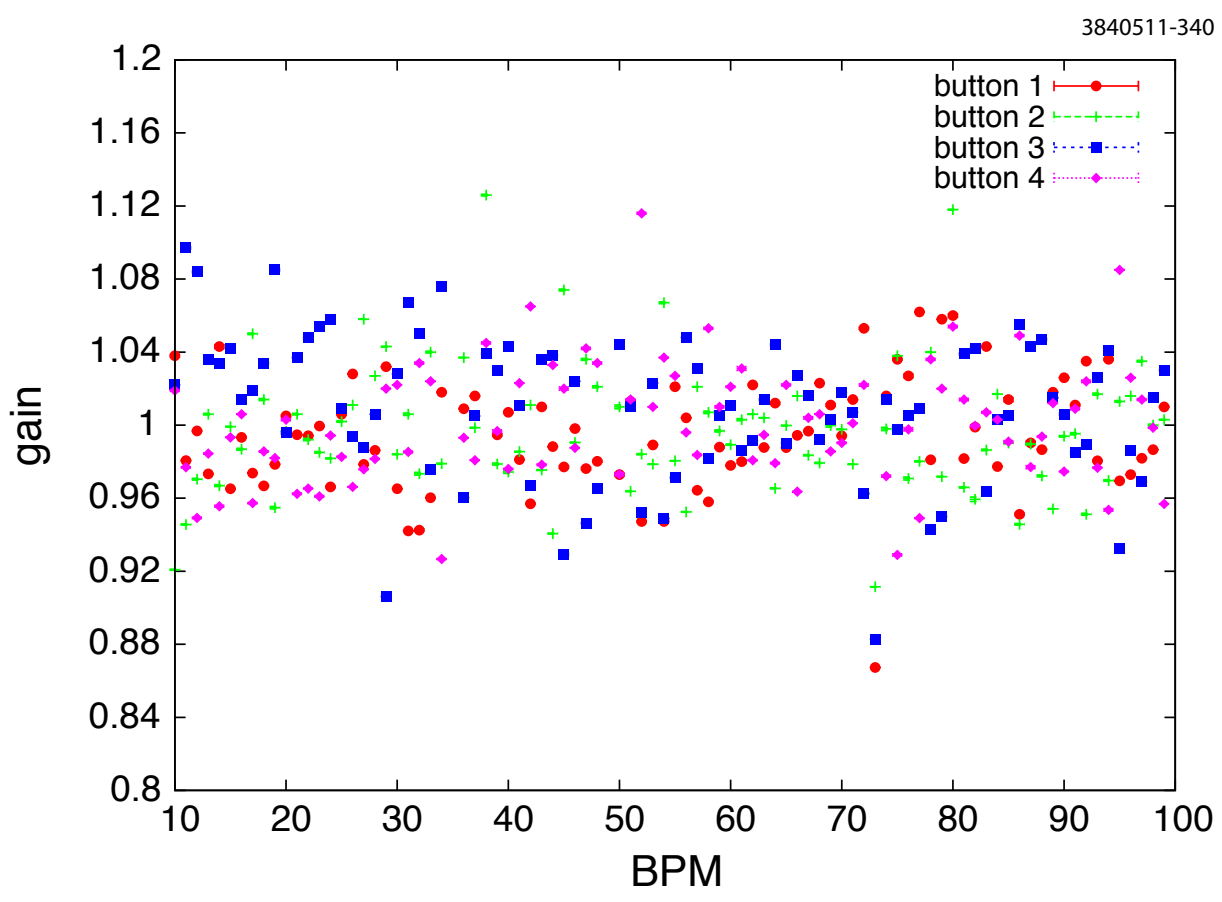

Figure 3. Measured BPM button gains. Note that there are four data points for each BPM.

A histogram of the individual gains is shown in left-hand plot of Figure 4. The accuracy of the beam based method for determining button gains is reflected in the reproducibility of the fitting procedure. The right-hand plot of Figure 4 shows the distribution of the variation in fitted gains for 
seven distinct sets of turn-by-turn data. From this one can conclude that the gains are characterized to within a fraction of a percent precision.
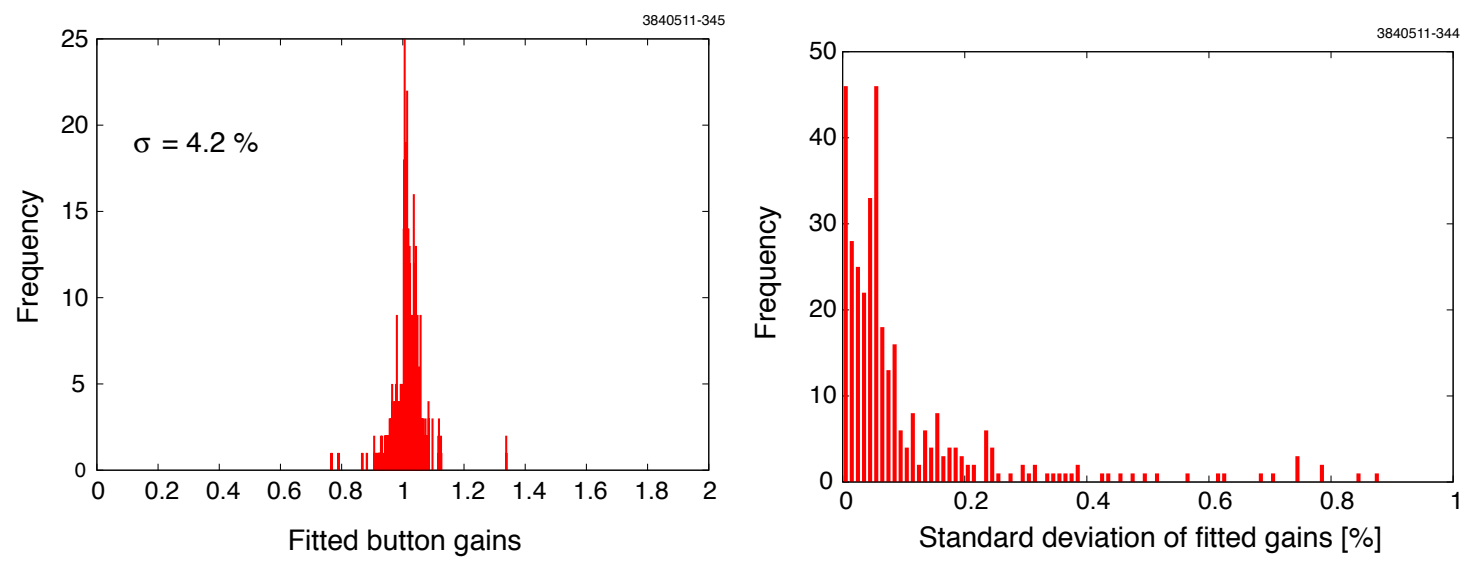

Figure 4. (Left) Distribution of measured button gains, with standard deviation 4.2\%. (Right) Distribution of the variation in fitted button gains for seven sets of turn-by-turn data.

\subsection{BPM Tilts}

As described above BPM tilts couple measured horizontal and vertical dispersion. In particular for a BPM horizontal-to-vertical tilt $\theta, \Delta \eta_{y} \sim \eta_{x} \theta$. Peak horizontal dispersion in the CesrTA lattice is about 1-2 m. In order that the error in the measured vertical dispersion be less than $10 \mathrm{~mm}$, we require that uncertainty in the BPM tilts be less than $10 \mathrm{mrad}$. BPM tilts can be extracted from the coupling measurements by taking advantage of the fact that the out of phase component of the coupling matrix $\bar{C}_{12}$ is insensitive to tilt. Then if $\bar{C}_{12}$ is corrected with skew quads, the in phase components $\bar{C}_{11}$ and $\bar{C}_{22}$ provide a direct measure of the physical tilt.

The algorithm for extracting BPM tilt from the coupling data is as follows:

1. Measure and correct coupling based on $\bar{C}_{12}$

2. Remeasure coupling, specifically $\bar{C}_{11}$ and $\bar{C}_{22}$.

3. Model the measured $\bar{C}_{11}$ and $\bar{C}_{22}$ by fitting BPM tilts.

The procedure has been applied to 58 sets of coupling data, all collected during the course of the June 2011 run. The 58 data sets include measurements in five distinct lattice configurations and at four different beam energies. The average along with the rms of the residuals of the fitted tilts are shown in left-hand plot of Figure 5. A histogram of the RMS of the residuals is shown in right-hand plot of Figure 5. Uncertainty in the measured tilts is less than 10 mrad.

\section{Performance of Low Emittance Instrumentation}

\subsection{Orbit}

We estimate the intrinsic resolution of the orbit measurement in terms of reproducibility. The variation in the measured positions for twenty consecutive measurements is shown in Figure 6. 

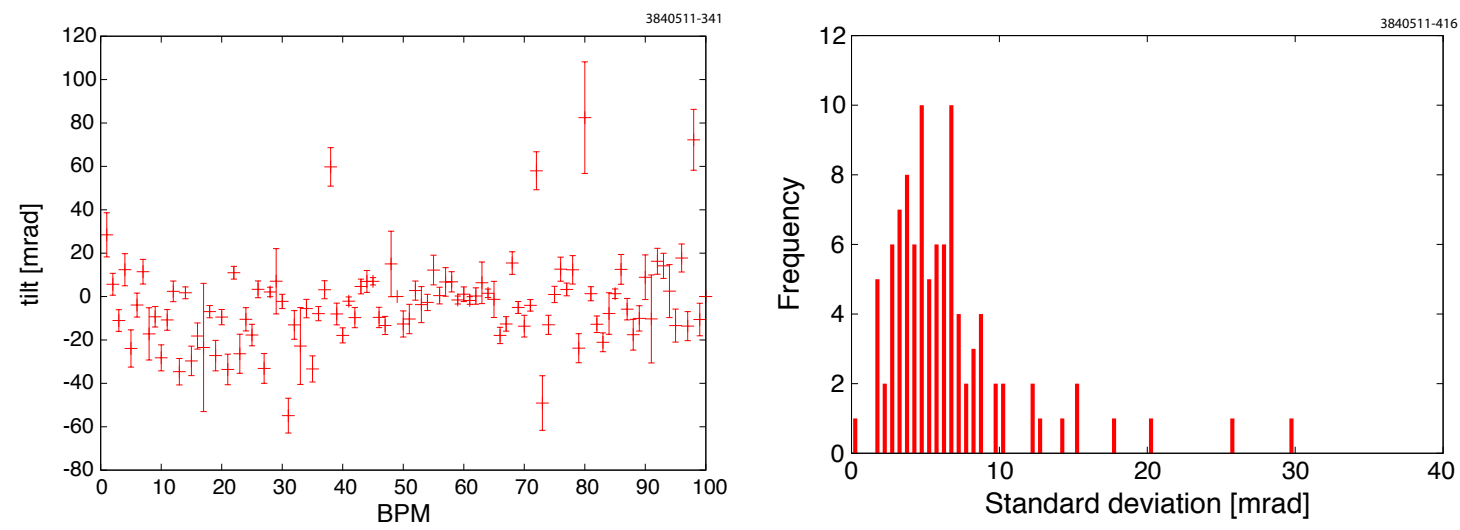

Figure 5. (Left) Measured BPM tilts. The error bars are $\sigma=\frac{1}{N} \sqrt{\sum_{i=1}^{N}(\theta-\langle\theta\rangle)^{2}}$ of 58 data sets. (Right) The distribution of the RMS residuals.

The standard deviation of horizontal and vertical position measurements is $7.8 \mu \mathrm{m}$ and $5.8 \mu \mathrm{m}$, respectively. This does not follow the expected $1 / \sqrt{N}$ behavior for random noise, which implies true beam motion is contributing to the orbit measurement resolution. The source of this beam motion is not diagnosed at the time of this publication, and efforts continue on this issue.
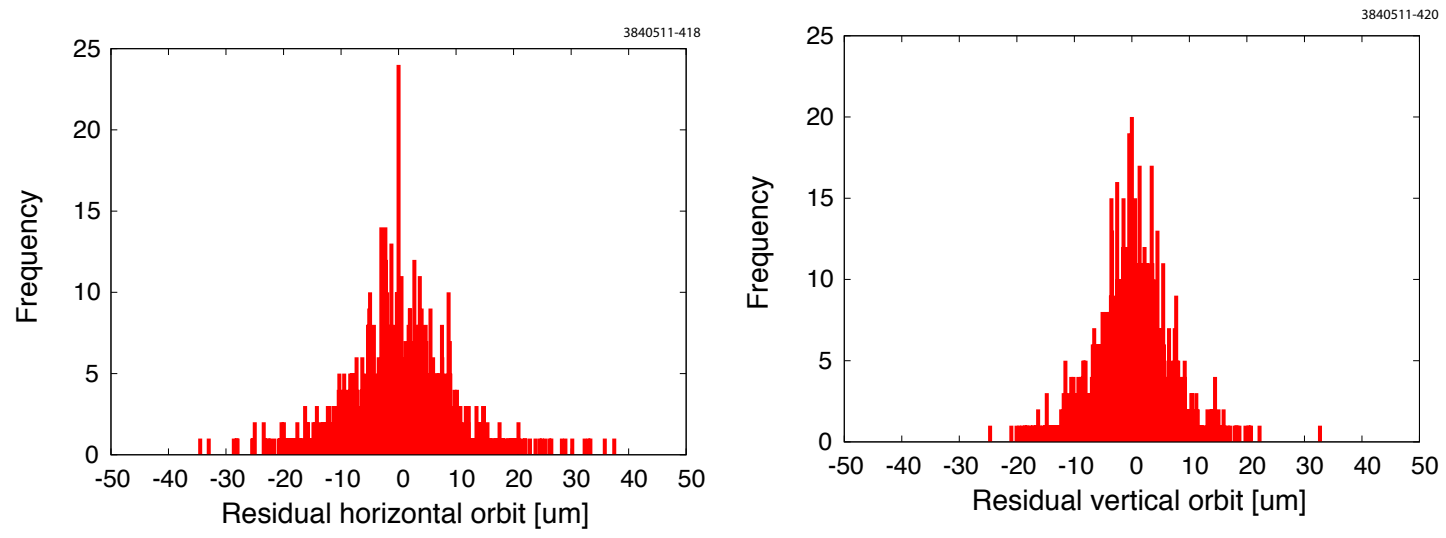

Figure 6. Reproducibility of horizontal (left) and vertical (right) orbit measurements. The standard deviation of the measurements is $7.8 \mu \mathrm{m}$ and $5.8 \mu \mathrm{m}$, respectively.

\subsection{Dispersion}

The distribution of the variation of the measured horizontal and vertical dispersion is shown in Figure 7. The standard deviations are $4.6 \mathrm{~mm}$ for horizontal and $2.3 \mathrm{~mm}$ for vertical dispersion, corresponding to the intrinsic resolution of the dispersion measurement. The accuracy of the dispersion measurement is limited by the systematics described above, rather than the intrinsic resolution of the beam position monitors. 

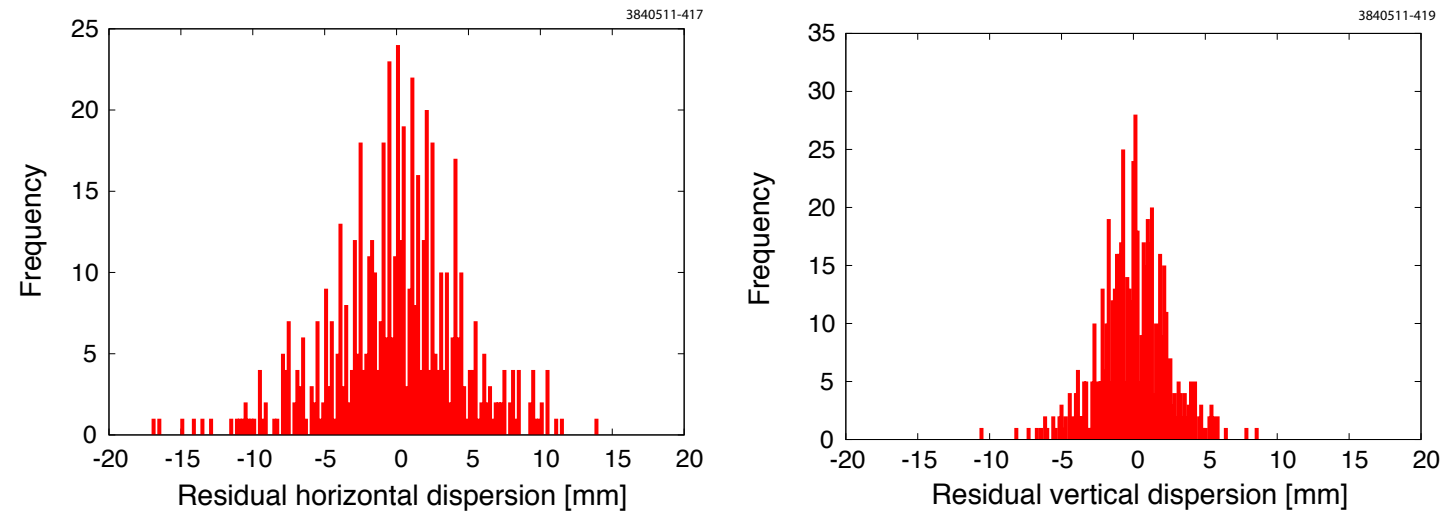

Figure 7. Reproducibility of horizontal (left) and vertical (right) dispersion measurements. The standard deviation of the measurements is $4.6 \mathrm{~mm}$ and $2.3 \mathrm{~mm}$ respectively.

\subsection{Phase and Coupling}

In order to determine the intrinsic resolution limit of the phase and coupling measurement, we repeat the measurement 20 times consecutively. Differences from one measurement to the next are due either to drift in machine parameters or statistical limitations of the technique. The distribution of the residuals with respect to the average measurement of vertical phase for each BPM is shown in the top plot of Figure 8, and the distribution of residuals with respect to the average coupling $\left(\bar{C}_{12}\right)$ are shown in the bottom plot of Figure 8.
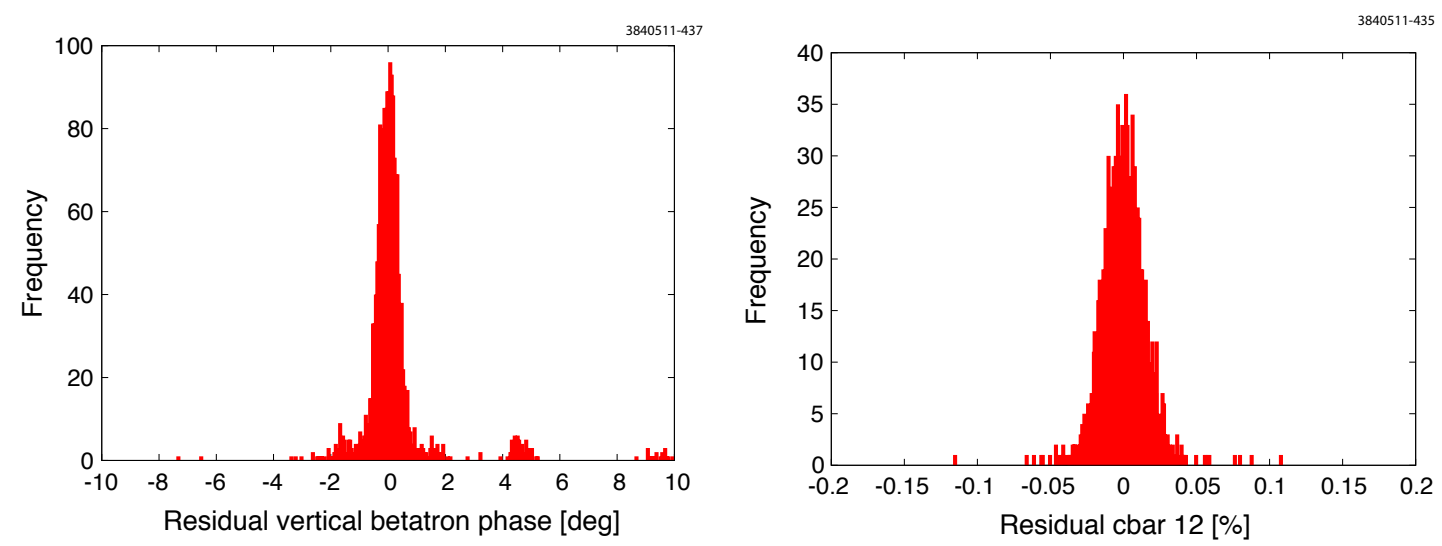

Figure 8. Reproducibility of vertical betatron phase measurements (left) and transverse coupling element $\bar{C}_{12}$ (right).

\section{4 xBSM - Vertical Beam Size}

The xBSM images synchrotron radiation $\mathrm{x}$-rays emitted in a hard bend dipole on a bunch-by-bunch, turn-by-turn basis using a linear array of 32 photodiodes [17]. The xBSM is the principle tool for measuring vertical emittance and determining the effectiveness of the low emittance tuning procedures. 
Several optics are available for the xBSM. A simple pinhole image is the most straightforward to interpret and is the basis for most of the low emittance tuning studies. (The optic that is described as the "pinhole" is in reality a vertical gap with adjustable height.) Neglecting diffraction, light from a point source passing through a slit of height $G$ produces an image of width $\sigma_{\text {pointsource }}=\frac{G}{x_{s}}\left(x_{i}+x_{s}\right)$, where $x_{i}$ and $x_{s}$ are distances from optic-to-image and source-to-optic, respectively. The "effective height" of the pinhole can then be taken to be $\sigma_{p}=\sigma_{\text {pointsource }} / M$, where $M=x_{i} / x_{o}$ is the magnification of the pinhole. The pinhole (gap height) is set to give minimum image size for the mean x-ray energy of $2 \mathrm{keV}$ (at $2.085 \mathrm{GeV}$ beam energy). The effective height, $\sigma_{p}=21 \pm 2 \mu \mathrm{m}$, is defined as the convolution of magnified gap image and diffraction distribution.

The final beam image will be computed as the convolution of the image of the source from an infinitely small pinhole and the image of a finite-height gap from a point source. Details of how the xBSM data is post-processed into a beam size are available in [17]. Systematic and statistical error analysis is shown in $[6,7]$.

\subsection{Summary of Low Emittance Measurement Resolution}

The resolution of measurements of beam position, dispersion, coupling, betatron phase advance, and vertical beam size are derived in [6, 7], and are summarized in Table 1.

Table 1. Accuracy of beam based measurements. Vertical emittance resolution is detailed in [7]

\begin{tabular}{|c|c|}
\hline Parameter & RMS \\
\hline BPM position (absolute) $[\mu \mathrm{m}]$ & 170 \\
BPM position (differential) $[\mu \mathrm{m}]$ & 10 \\
Dispersion $[\mathrm{mm}]$ & 10 \\
Coupling $\left(\bar{C}_{12}\right)[\%]$ & 0.2 \\
Betatron phase $[\mathrm{deg}]$ & 0.1 \\
Vertical beam size $[\mu \mathrm{m}]$ & 2.5 \\
Vertical emittance (statistical / systematic) $[\mathrm{pm}]$ & $\pm 3.5 / \pm 0.3$ \\
\hline
\end{tabular}

\section{Electron Cloud and Intra-beam Scattering Experimental Hardware and Tech- niques}

This section describes the measurement techniques that are required for EC dynamics and intra-beam scattering measurements in CesrTA. The requirements for the single-bunch intra-beam scattering are generally a subset of those employed for the multi-bunch EC dynamics measurements. (One notable exception to this is that intra-beam scattering measurements include streak camera measurements for determining bunch lengthening and these are not routinely employed for EC dynamics measurements.) As a result this section will focus on the techniques for EC dynamics measurements exclusively with the understanding that many of these will also be utilized for intra-beam scattering measurements. 


\subsection{Requirements for Electron Cloud Induced Beam Studies}

One of the key goals of the CesrTA research program has been to improve the understanding of the interaction of the EC with the high energy particle beam. The initial motivation for this was to allow the extrapolation from the experimental conditions of CesrTA to the conditions anticipated for the ILC damping rings.

The interaction of the particle beam with the cloud can be studied by measuring the properties of the beam in the presence of the cloud. The key beam properties which are influenced by the cloud are the beam's closed orbit distortion (quite small, and not extensively studied with CesrTA), the frequency spectrum of the beam centroid's coherent dipole motion relative to this orbit and the beam's transverse position distribution.

In CesrTA, the beam is configured longitudinally into a train of $\sim 10 \mathrm{~mm}$ long bunches separated by an adjustable spacing (variable from a minimum of $4 \mathrm{~ns}$, up to a maximum equal to the revolution period, $2.56 \mu \mathrm{s}$ ). For sufficiently closely-spaced bunches, the EC grows along the train and so the cloud environment is different for each bunch. For this reason, it is critical that the beam dynamics measurement made to probe the cloud be done on a bunch-by-bunch basis.

The frequency spectrum of the coherent motion of each bunch contains a wealth of information. In particular, this information includes

- the amplitude, frequency, and line shape of the betatron dipole lines, which are sensitive to the electron cloud's electric field, to the mode of oscillation of the bunches in the train, and to the presence of multi-bunch instabilities;

- the amplitude, frequency, and line shape of head-tail lines, which are generally separated from the betatron lines by approximately the synchrotron frequency, and are sensitive to internal motion within the bunch driven by EC-induced single-bunch head-tail instabilities.

In addition after bunch motion has been excited by an external source, the time dependence of the amplitudes of the betatron dipole and head-tail lines provide information on the damping of these lines, which is related to aspects of the effective EC impedance that is not probed by tune measurements. In the sections below the experimental hardware and techniques used to obtain the measurements are described and the some typical beam dynamics observations are presented.

There are several beam parameters particularly relevant for the study of electron cloud effects. Since the EC can produce focusing of the stored beam, measuring the betatron tunes of bunches along the train gives information about the average density of the cloud along the length of the train. The electron cloud can also produce unstable motion in later bunches in the train. To observe the unstable motion, it is necessary to detect the amplitude at the betatron frequency and any other frequencies representing different modes of oscillation (e.g. head-tail modes) of bunches within the train. The unstable motion may also result in enlargement of the vertical beam size, thus the measurement of the vertical beam size for each bunch in the train is important. Before beam conditions approach the regime for the onset of unstable motion, it is possible to measure the damping of coherent motion of the bunches using drive-damp techniques. This method excites coherent dipole betatron modes or head-tail modes for each bunch within the train and then observes the damping of the motion. Thus it is possible to observe how the coherent motion becomes less 
stable before the onset of instability. The methodology and examples of typical measurements for these techniques are presented in the following sections.

\subsection{Bunch-by-Bunch Tune Measurements}

During the course of the CesrTA project several different techniques have been utilized for making tune shift measurements for individual bunches within trains of bunches. These techniques, their benefits and their limitations will be described in this section.

\subsubsection{Multi-bunch Large Amplitude Excitation}

This method for observing the tunes of different bunches within the train consists of pulsing a pinger magnet with a single-turn excitation to deflect all of the bunches within the train, thereby starting a dipole oscillation of their centroids. The CBPM system is then timed to read out a number of BPMs over several thousand turns for all bunches in the train; the data acquisition is synchronized with the triggering of the pinger magnet's deflection. Turn-by-turn trajectory data are recorded, typically for 8192 turns. Note that the damping time depends on a bunch's position within the train; bunches at the start of the train have transverse damping times of 10-20,000 turns, whereas bunches later in the train have shorter damping times due to their interaction with the electron cloud. The data are analyzed offline with a Fast Fourier transform (FFT) algorithm, from which the betatron tunes are determined. For 8192 turns, the FFT has frequency resolution $f_{\text {rev }} / 8192=390.1 \mathrm{kHz} / 8192=48 \mathrm{~Hz}$; more advanced methods have improved resolution. During many of these measurements the peak horizontal and vertical beam displacements were typically $5 \mathrm{~mm}$ at $2.1 \mathrm{GeV}$ and $2 \mathrm{~mm}$ at $5.3 \mathrm{GeV}$.

Since data from all bunches is recorded at the same time, it is relatively rapid to take data in one set of conditions and, since the data from all bunches is taken on the same turns, this method is relatively insensitive to any drifts in the storage ring tunes. However, the fact that all bunches are excited at the same instant implies that the lowest coupled-bunch mode is necessarily excited for the train of bunches. When the train is oscillating in this mode, the bunch-by-bunch horizontal tune shifts induced by the EC are strongly suppressed, and difficult to measure. It is also the case that the pinger excitations are relatively large with respect to the stored beam's size: e.g., typically the vertical oscillation amplitude may exceed several ten's of vertical sigma. So the beam's oscillation is exploring a fairly large volume of the electron cloud charge distribution.

\subsubsection{Single Bunch Small Amplitude Excitation}

Another approach has been developed for bunch-by-bunch tune measurements. This approach excites only a single bunch in the train, thereby reducing the coupling from earlier bunches to the bunch that is being measured. This is accomplished by driving both the horizontal and vertical stripline kickers (shown schematically for one stripline kicker in Fig. 9), using the external modulation input for the beam stabilization feedback system, which allows gating of the input signal into the appropriate timing window in order to excite only the bunch being measured. The source for the signal for the external modulation port comes from a frequency synthesizer, whose output frequency is swept across the range of betatron oscillation frequencies, covering the tunes for the entire ensemble of bunches. The frequency is swept with a saw-tooth at $500 \mathrm{~Hz}$, driving the bunch 
in its dipole oscillation mode when the excitation frequency crosses the betatron resonance. The turn-by-turn position data are recorded for a number of BPMs using the CBPM system readout with the total number of turns sufficiently large to capture at least one full excitation and damping cycle. The measurement process is repeated as the excitation's delay is stepped from one bunch to the next, resulting in a set of positions for all bunches at each delay. The data are analyzed offline with an FFT to give the oscillation frequency of the excited bunch and coupling of its motion to subsequent bunches via the EC.

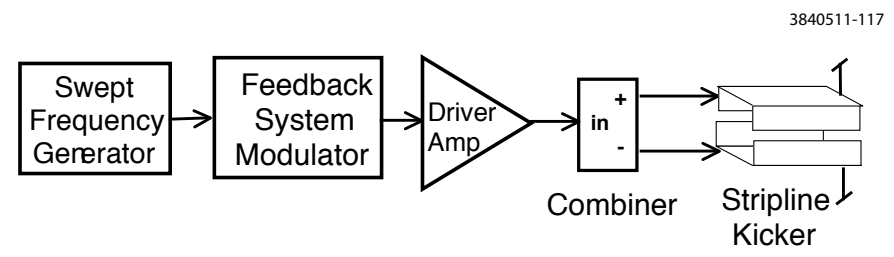

Figure 9. Single bunch excitation method using the stripline kicker, driven by a swept frequency source via the feedback system's external modulation port.

Some results are presented here to illustrate this technique; the data were taken with a 10 bunch train having a $14 \mathrm{~ns}$ spacing in $2.1 \mathrm{GeV}$ beam energy conditions. Fig. 10 shows the horizontal position data for the fourth, fifth and sixth bunch, when only bunch number 5 was being excited. During the 2048-turns of the data-samples taken on simultaneous turns for the three bunches, it is clear that bunch 5 was excited with two complete cycles of the swept signal source. This is even clearer in Fig. 11 which shows the horizontal spectra of all 10 bunches when bunch numbers 1, 5 and 10 were being driven individually. The fact that the stripline kicker is driving only one bunch is quite evident in both Figures 10 and 11.

For comparison with the horizontal data, the matching set of vertical data are presented here for the same storage ring and EC conditions as above. The vertical position data for bunches 4,5 and 6 is shown in Fig. 12, when only bunch 5 is driven. Also the vertical spectra for all bunches are shown in Fig. 13, when bunches 1, 5 and 10 are individually excited. An interesting feature, visible in the vertical data, is that even though only one bunch is being driven, its motion couples to subsequent bunches in the train. Fig. 13 presents evidence that this coupling increases along the train, suggesting that the EC may be playing a role in this bunch-to-bunch vertical dipole coupling.

This technique has the advantage of avoiding coupling from preceding bunches to the bunch being studied, while also providing information about the coupling of the motion of one bunch to later bunches via the EC. The excitation level can, in principle, be tailored for the bunch that is being driven; the ability to keep a relatively fixed oscillation amplitude of the driven bunch could be important for conditions when the first bunches in the train are more stable but the latter bunches are not. This method has the drawback that it is slower than the preceding method, as it requires collecting turn-by-turn position data for every bunch times the number of bunches within the train. It is, therefore, sensitive to drifts in the tunes of the storage ring. The data acquisition software is sufficiently flexible to permit the excitation of the first bunch in the train (which generally has quite weak coupling due to the EC to all of the other bunches in the train) in addition to the bunch actually being studied. In this way the tunes of the later bunches are measured with respect to the 


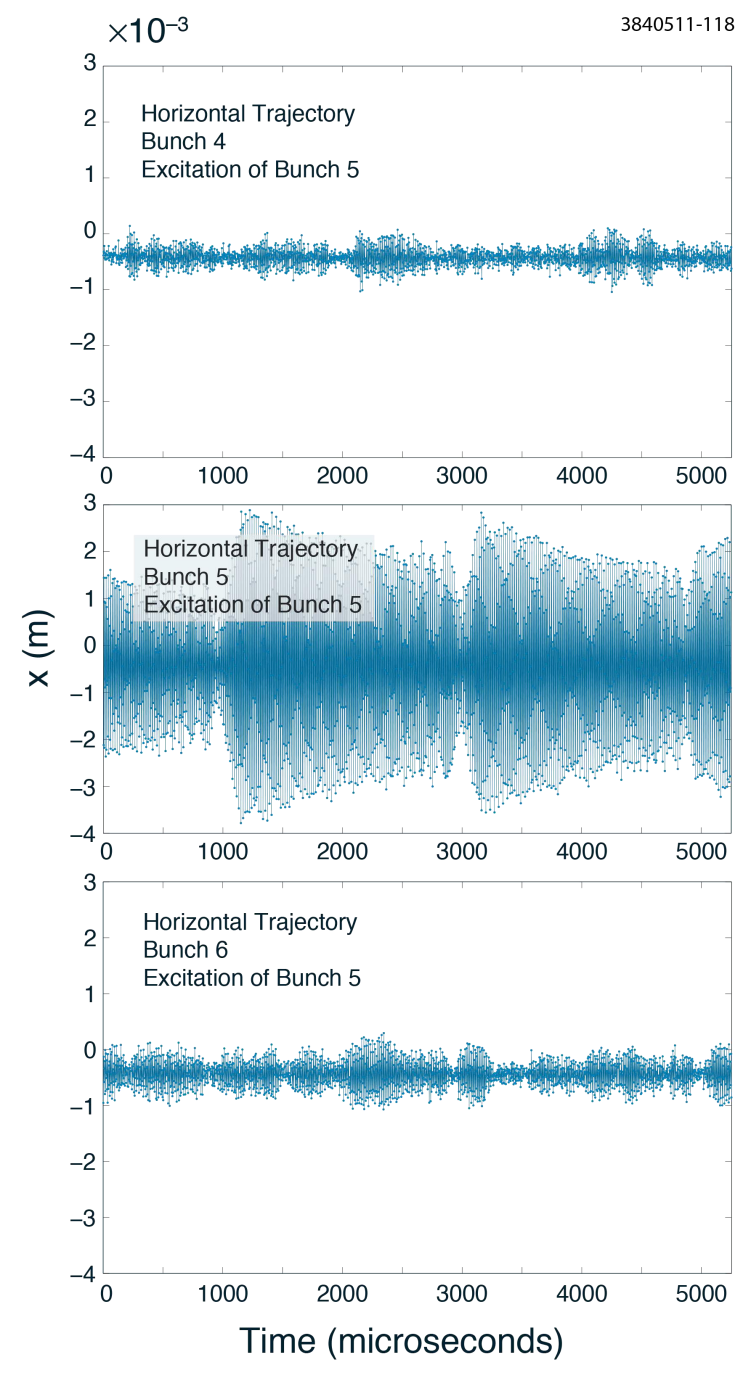

Figure 10. Horizontal position of bunches 4, 5 and 6 (respectively for the top, middle and bottom plots) for a 10-bunch train when only bunch number 5 was excited.

tune of the first bunch, which allows for correction of drifts in the tune during the measurement. Another feature is the ability to turn off feedback for the bunches which are being excited. This permits the train of bunches to be stabilized with feedback, while allowing the bunches, which are being measured, to have longer damping times, permitting a more accurate tune measurements and the ability to measure the variation of damping rates of different bunches within the train.

\subsubsection{Feedback System Response}

Another approach for tune measurements became apparent after the installation of the Dimtel[37] feedback electronics, capable of damping bunches with spacings down to $4 \mathrm{~ns}$. While looking at the FFT of the position for a single bunch as part of the feedback system diagnostics, it was observed that the signal amplitude varied as a function of the feedback gain. At low gains the betatron peak is visible, but as the gain is increased the amplitude of the peak decreases until it become a notch in 


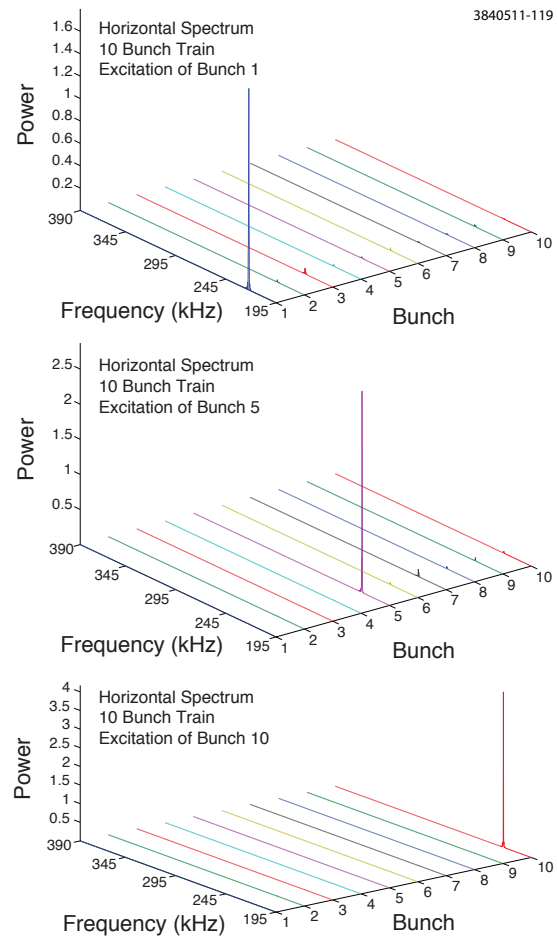

Figure 11. Horizontal position spectra of all bunches in a 10-bunch train when bunches number 1, 5 and 10 (respectively for the top, middle and bottom plots) were driven individually.

the spectrum at high gain. The notch is created by the feedback system, whose phase is adjusted to suppress the broadband excitation of the beam preferentially at the betatron frequency. When the feedback settings have been fully optimized, the notch in the spectrum for each bunch marks the location of its betatron oscillation frequency.

The position data generally represents the effect of probing the EC in a regime when the bunches are moving at small amplitudes. An example of data taken using this method is seen in Fig. 14. There is a very clear trend for the vertical focusing effect from the accumulating EC, which is visible the plot. Although this method is quite appealing, only a few tune shift measurements have been performed via this method. This technique works well for 4 ns-spaced bunches, but it requires fairly exact adjustments of the feedback system parameters to be able to clearly identify the notches in the bunch spectra. To obtain the most accurate spectra, the data for each bunch is averaged typically for 30 seconds, allowing some variation in the tunes due to longer-term drifts in the storage ring focusing.

\subsubsection{Self-Excitation}

The last method utilized for bunch-by-bunch tune shift measurements is a by-product of the observation of beam instabilities, described in the next section. In this set of measurements the position spectrum of each bunch is measured with a gated spectrum analyzer. Two of the peaks that are visible in these self-excited spectra are the horizontal and vertical dipole modes. The shift of the tunes as the gate is moved from bunch to bunch are easily detected via this method. Since most of 


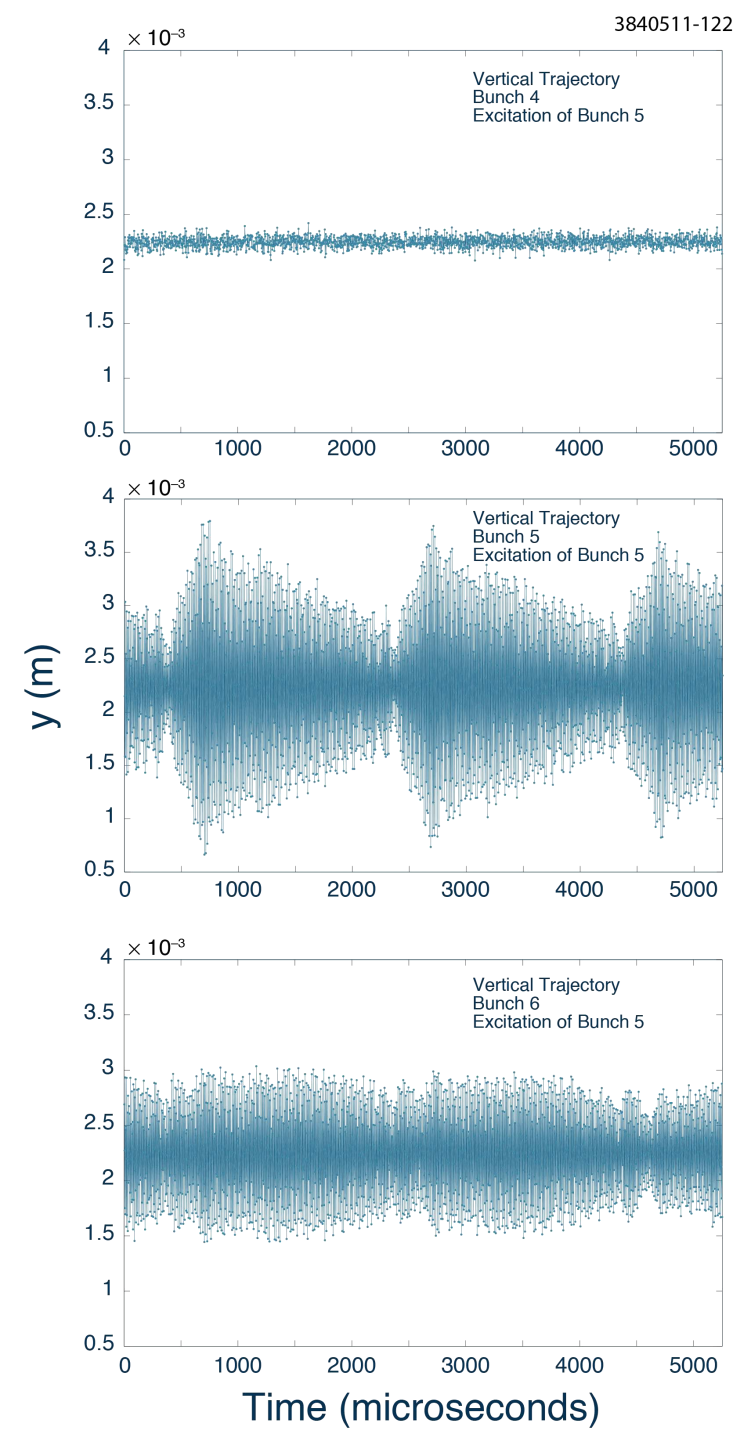

Figure 12. Vertical position of bunches 4,5 and 6 (respectively for the top, middle and bottom plots) for a 10-bunch train when only bunch number 5 was excited. Turn-by-turn position indicated by red dots, connected by blue lines.

these measurements are taken in conditions when the beam is above or near an instability threshold for at least some of the bunches within the train, the self-excited amplitudes of the dipole motion will vary along the train. This method is quite sensitive to low signal levels, with the noise floor for small amplitude oscillations at the level of $0.4 \mu \mathrm{m}$-rms horizontally and $0.2 \mu \mathrm{m}$-rms vertically. Due to averaging in the spectrum analyzer, the data acquisition requires about 1 minute for each bunch, which is long enough to make this method sensitive to drifts in the storage ring tunes.

\subsection{Instability Measurements}

An important set of CesrTA measurements focuses on beam instabilities due to the EC. These studies measure the growth of self-excited oscillation amplitudes of the bunch centroids and the 

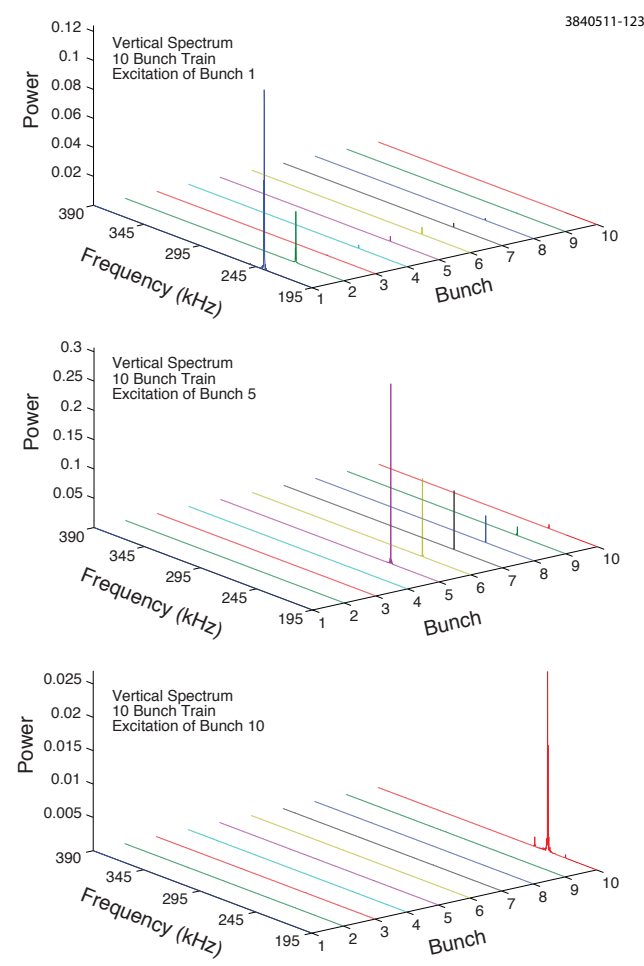

Figure 13. Vertical position spectra of all bunches in a 10-bunch train when bunches number 1,5 and 10 (respectively for the top, middle and bottom plots) were driven individually.

3840511-097

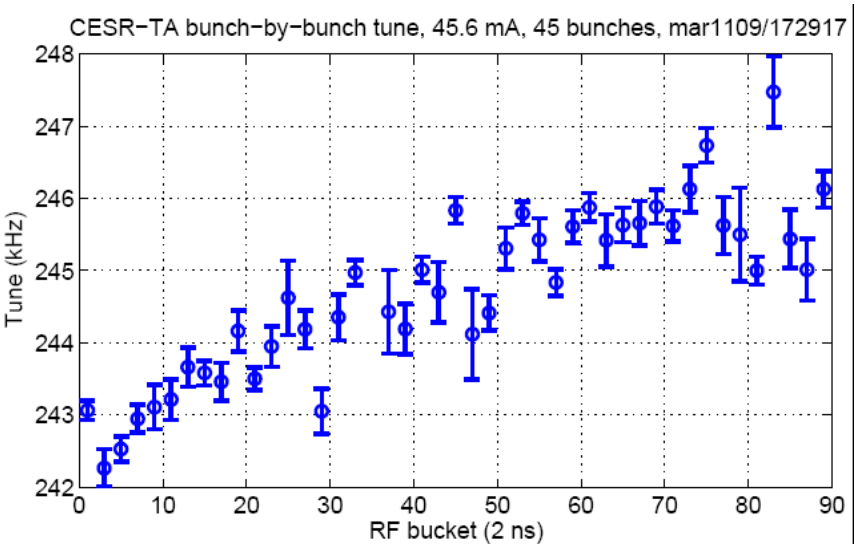

Figure 14. Vertical tune vs. RF bucket number for a train of 45 bunches with $4 \mathrm{~ns}$ bunch spacing determined from notches in the spectra from the feedback error signal. 
growth of vertical beam size along the train under various accelerator and electron cloud conditions. The first piece of hardware utilized for these measurements is a monitor for the bunch-by-bunch beam position. The other detection system required is the XBSM monitor for determining the vertical beam size of each bunch.

\subsubsection{Bunch-by-Bunch Position Spectra}

For instability studies, the bunch-by-bunch position measurements are accomplished by a BPM detector connected to one of CESR's original relay-based BPM system processors, which in turn passes its video output signal to a spectrum analyzer in the control room. (See reference [4] for the description of the hardware.) BPM33W, which is located at a high vertical beta point, has generally been used as the detector for these observations. The signal is taken from one button, making it sensitive to both the horizontal and vertical motion. The data-taking software sets the trigger delay for the sampling gate to select a particular bunch within the train. For almost all of the data, an RG-174 coaxial cable is placed within the signal path to limit the bandwidth of the button signal (giving an effective $20 \mathrm{~dB}$ of signal attenuation) and to this an additional $12 \mathrm{~dB}$ of amplification is added. The signal is then sent to the biased peak rectifier circuit, which has an effective bandwidth of $700 \mathrm{MHz}$, and a decay time constant of approximately $5 \mu \mathrm{s}$. The resulting video signal is buffered and sent on a wide-band coaxial cable to a spectrum analyzer in the control room.

The spectrum analyzer is a Hewlett Packard model 3588A, operating in the baseband (in these studies the center frequency ranges from $190 \mathrm{kHz}$ to $310 \mathrm{kHz}$ ) in "Narrowband Zoom" mode with a $40 \mathrm{kHz}$ span. This mode of operation performs a $\pm 20 \mathrm{kHz}$ FFT on time slices of the signal and these spectra are averaged for 100 time slices, taking about 10 seconds for each $40 \mathrm{kHz}$ step of the center frequency. At $2.1 \mathrm{GeV}$ the position sensitivity of the signal from the BPM at $33 \mathrm{~W}$ was measured to be

$$
\begin{aligned}
& x_{\mathrm{rms}}=x_{0} / I_{b}(\mathrm{~mA}) \times 10^{A_{x}(\mathrm{dBm}) / 20} \\
& y_{\mathrm{rms}}=y_{0} / I_{b}(\mathrm{~mA}) \times 10^{A_{y}(\mathrm{dBm}) / 20}
\end{aligned}
$$

where $x_{0}=81.3 \mathrm{~mm}$ and $y_{0}=45.3 \mathrm{~mm}$, when the RMS bunch length was approximately $10 \mathrm{~mm} . A_{x}$ and $A_{y}$ are the amplitudes measured on the spectrum analyzer in $\mathrm{dBm}$. With this gain configuration and over the frequency range of study, the noise baseline falls from $-95 \mathrm{dBm}$ to $-105 \mathrm{dBm}$ (corresponding in the vertical direction, respectively, to displacements of $1.1 \mu \mathrm{m}$-RMS to $0.33 \mu \mathrm{m}$ RMS for a $1 \mathrm{~mA}$ bunch.)

Representative self-excited spectra of the first and last bunch in a 30-bunch positron train at $2.1 \mathrm{GeV}$ are shown in Figures 15 and 16. For this train the horizontal tunes are in the range from $212 \mathrm{kHz}$ to $218 \mathrm{kHz}$, and the vertical tunes are in the range from $224 \mathrm{kHz}$ to $227 \mathrm{kHz}$. Since this spectrum overlaps the $1 / 2$ integer resonance at $195 \mathrm{kHz}$, this frequency is a reflection point for the spectra. For bunch 30, additional lines are visible in the ranges $198-201 \mathrm{kHz}$ and $250-252 \mathrm{kHz}$; these correspond to vertical head-tail modes as their frequencies are plus and minus the synchrotron oscillation frequency added to the vertical tune. The baseline is seen to be falling as roughly a $1 / f$ noise spectrum. There are also a number of unrelated noise lines, scattered throughout the spectra assumed to be due to "cultural noise sources." A "mountain-range" plot of the spectra of all 30 bunches within a 30 bunch-long train is shown in Figure 17. A cut of the spectrum has been 
made at the half integer resonance $(195 \mathrm{kHz})$ to suppress the "reflected" spectral lines. In this plot the self-excited vertical tune amplitude begins to grow at approximately bunch 10 and continues to grow in amplitude until near bunch 20. In this region the two vertical head-tail lines appear above the noise background. Also around bunch 15 the spectral peak of the horizontal tune appears to bifurcate, something which is also seen in Figure 16, and on close examination these data also show bifurcation of the vertical tune and the vertical head-tail lines for the last bunches in the train. Figure 17 also shows a number of "fences", i.e. peaks in the spectrum at fixed frequencies due to external "cultural noise sources."

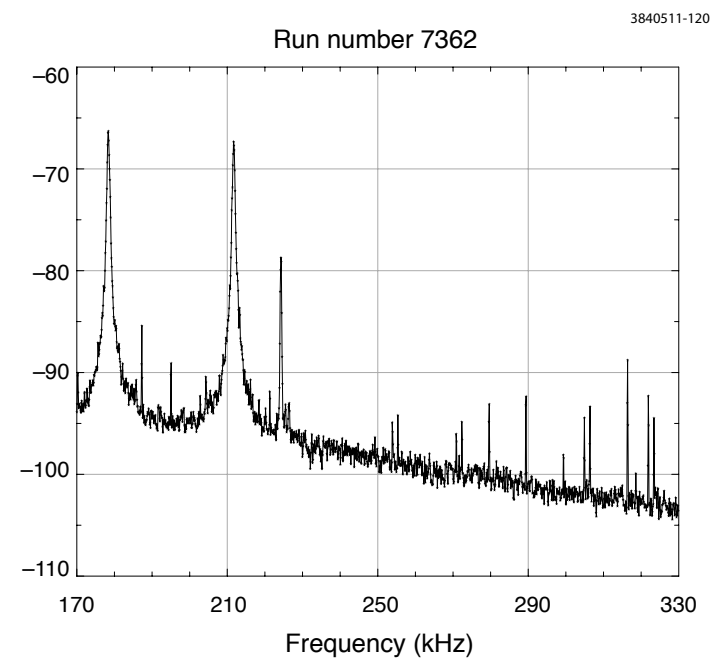

Figure 15. Self-excited beam power spectrum for bunch 1 in a 30 bunch-long positron train at $2.1 \mathrm{GeV}$ beam energy. Since this spectrum overlaps the $1 / 2$ integer resonance at $195 \mathrm{KHz}$, this frequency is a reflection point for the spectra. Thus the peak at $178 \mathrm{KHz}$ is a reflection of the peak at $212 \mathrm{KHz}$.

Many tests have examined the self-consistency and interpretation of the data. The identification of the vertical and horizontal tunes was checked by changing the controls for each separately and verifying which spectral peak moved. They were also checked using BPMs at other locations, which had buttons summed to produce dominantly horizontally- or vertically-sensitive detectors. The interpretation that the vertical head-tail lines were not inter-modulation distortion components coming from the processing electronics was tested by switching a $6 \mathrm{~dB}$ attenuator into the signal path upstream of the peak detector and observing the change in both horizontal and vertical spectral peaks. If the head-tail lines were actually inter-modulation cross-products from the non-linearity of the electronic processing, then they would have decreased by $12 \mathrm{~dB}$, and they only decreased by $6 \pm 2 \mathrm{~dB}$.

Although this method for detecting the frequency spectra of the bunches is fairly sensitive, the measurements must be made separately for each individual bunch. The measurement time is about 1 minute per bunch for the selected frequency range. This means that the data represents a time-average of any unstable motion over this period. In addition, due to the finite beam lifetime, the beam must be refilled a number of times during data-taking for one set of conditions. In our case, we choose typically to refill after measuring spectra for five bunches. When these spectra are 


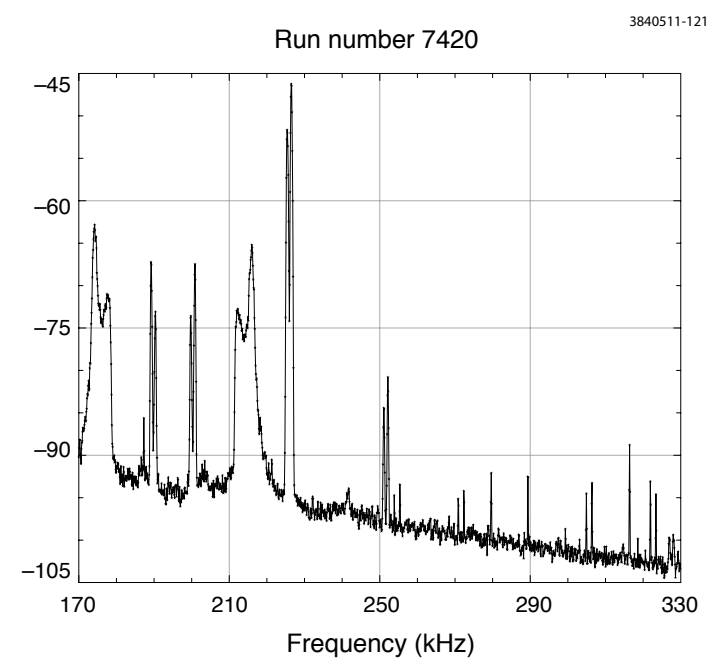

Figure 16. Self-excited beam power spectrum for bunch 30 in a 30 bunch-long positron train at $2.1 \mathrm{GeV}$ beam energy.

$3840511-102$

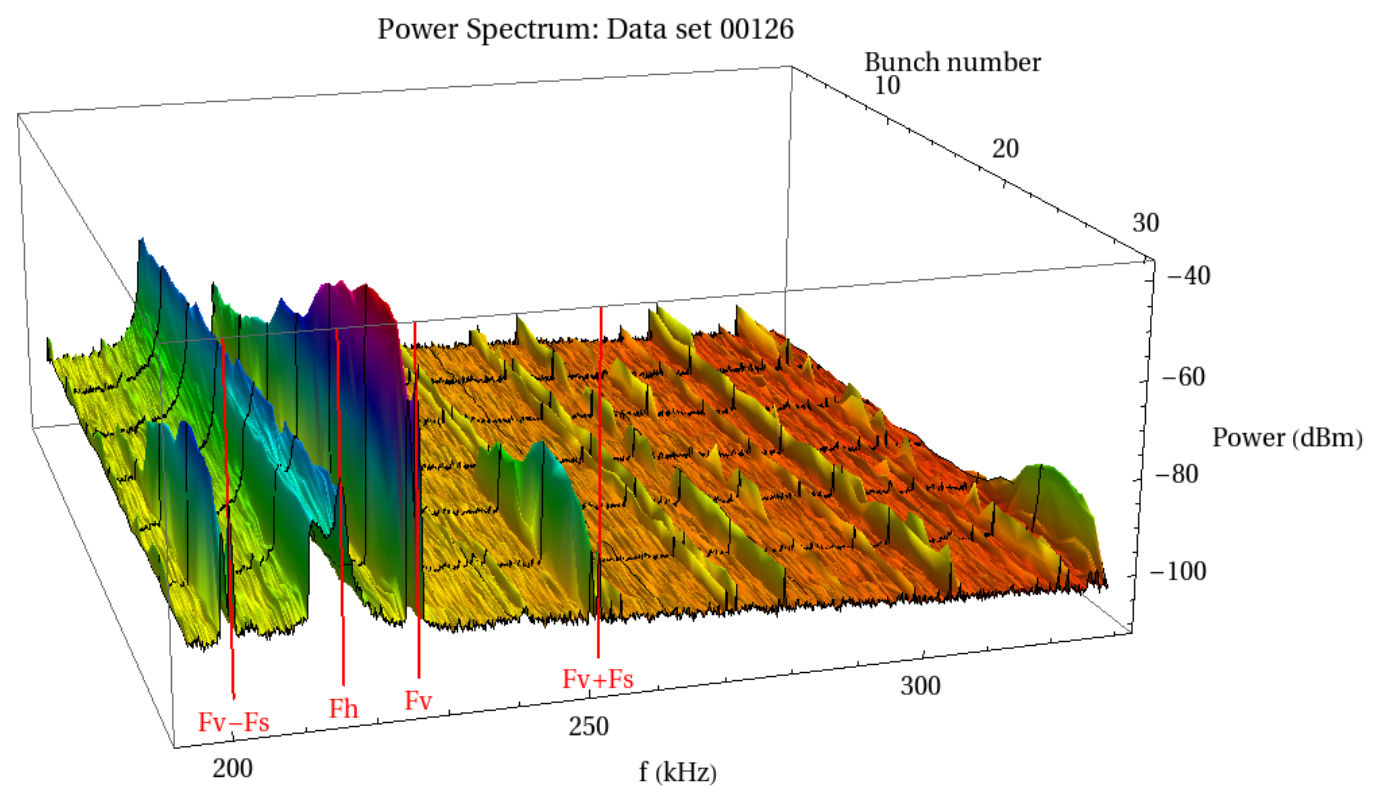

Figure 17. Self-excited beam power spectra for bunches 1 through 30 in a 30 bunch-long positron train at $2.1 \mathrm{GeV}$ beam energy. The horizontal axis is the frequency, the vertical axis is the spectral power in $\mathrm{dB}$ and the axis into the page is the bunch number with bunch 30 being in the foreground. Red vertical lines in the foreground denote in ascending order the location of the $m=-1$ vertical head-tail line, the horizontal tune, the vertical tune and the $m=+1$ vertical head tail line. 
plotted, the beam intensity decay over five bunches gives the amplitude for the peaks within the spectrum a slightly scalloped shape. This refilling cycle coordinates fairly well with the cycle to measure and readout the bunch-by-bunch and turn-by-turn XBSM data.

It is possible to compare the above measurements to those read out turn-by-turn and bunchby-bunch from a number of BPMs via the CBPM system (which has a much faster data acquisition time). Unfortunately the head-tail lines are not visible above the noise floor in the CBPM data. Our explanation is that the relay BPM system peak rectifies the position signal and, if there is a temporal variation due to head-tail motion, the arrival time of the signal varies correspondingly. This gives a frequency modulation to the position signal when viewed by the spectrum analyzer. The CBPM processing is different: the signal is sampled at a fixed time corresponding to the positive peak of the button BPM pulse. Any variation in the arrival time produces only a second order variation in amplitude and, even if one moved the sampling time significantly off of the peak, it does not produce any observable signal at the head-tail line frequencies.

\subsubsection{Bunch-by-Bunch Beam Size}

At CesrTA, bunch-by-bunch beam sizes are measured using an x-ray monitor [17] built on the D Line of the CHESS light source for viewing positrons. (A similar line for viewing electrons is installed at the $\mathrm{C}$ Line, useful particularly for comparison measurements for electron beams vs. positron beams.) The detector can read out bunch-by-bunch, turn-by-turn signals at $14 \mathrm{~ns}$ or 4 ns spacing. Three sets of x-ray optics can be selected in the optics box: Coded Aperture (CA), Fresnel Zone Plate (FZP) and an adjustable slit. The coded aperture mask permits single-shot, photon-statistic-limited resolutions of $\sim 2-3 \mu \mathrm{m}$ at vertical beam sizes of less than $20 \mu \mathrm{m}$ [38], [39].

During a given set of instability measurements, typically XBSM data were taken using two sets of optics, the adjustable slit and the CA. This allows the greatest range of sensitivity for measurements of the vertical size and centroid motion of the beam. During the measurement cycle, the beam size data are taken bunch-by-bunch and turn-by-turn generally immediately after the train has been topped off, usually occuring after taking the frequency spectrum for every fifth bunch.

\subsection{Mode Growth Rates}

A complement to the instability measurements, described in the preceding section, are the damping rate measurements for the coherent transverse modes. The instability measurements easily record the large amplitude coherent signals as the bunches become unstable and ultimately limit due to non-linearities in the bunch dynamics. However, the damping measurements give information about the stability of the bunch at small amplitudes before the bunch goes unstable, the regime in which storage rings and damping rings will actually operate. These studies give some insight about how the beam instability begins developing from earlier to later bunches along the train.

\subsubsection{Drive-Damp Excitation: Method}

The basic idea for these observations is to employ the same relay BPM configuration as is used for the instability measurements. However, the spectrum analyzer's center frequency is adjusted to be at either the vertical betatron dipole-mode frequency or one of the vertical head-tail mode 
frequencies while the spectrum analyzer is configured to be in "Zero Span" mode. In this mode the analyzer functions as a tuned receiver with its display producing signal amplitude vs. time. The spectrum analyzer's tracking generator output is connected to the vertical feedback system's external modulation input. Aside from the spectrum analyzer's control settings, this is quite similar to the hardware configuration shown in Fig. 9. By adjusting the digital timing controls for the feedback modulator's external input, it possible to drive only one bunch, as long as the bunch spacing is greater than $6 \mathrm{~ns}$ (if the bunch spacing is $4 \mathrm{~ns}$, then the duration of the pulse on the beam stabilizing feedback system stripline kicker is long enough to deflect the bunch under study and also slightly deflect the following bunch). To permit the drive-damp modulation of the beam, there is one additional element added to the block diagram of Fig. 9. This element is a modulating gate for the spectrum analyzer's tracking generator signal. The modulator gate is timed with the spectrum analyzer's timing sweep to pass the tracking generator output for $3 \mathrm{~ms}$ at the beginning of the sweep and then to gate off its output until the start of the next sweep.

An illustration of the timing and the expected signal response are shown in Fig. 18. The red curve shows that the amplitude of the transverse excitation of the bunch vs. time is an impulse. The expected beam response initially grows during the driving impulse, usually reaching a saturated level, and then decays exponentially after the drive is switched off (shown in the logarithmic plot as a linear decrease vs. time). If the frequency of the spectrum analyzer tracking generator is tuned away from the bunch resonant frequency, the decaying response can have more than one frequency component, resulting in periodic oscillatory beats. So during the measurement it is necessary to make small tuning adjustments to the excitation frequency to produce the most exponential decay possible.

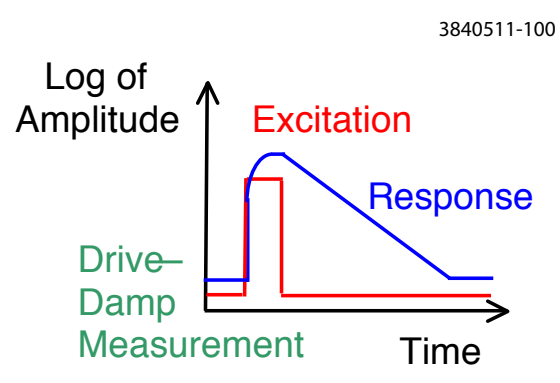

Figure 18. Illustration of the drive-damp measurement: The red trace is the amplitude of the excitation driving the bunch. The blue trace is the bunch response.

The excitation of the bunch is accomplished in a somewhat different manner for the betatron dipole mode and the head-tail modes. In both cases the frequency of the spectrum analyzer is set to drive the coherent mode frequency being measured. However, for the head-tail modes it is necessary to also continuously drive the external modulation input for RF cavity phase at the synchrotron oscillation frequency. A basic explanation is provided here, but more detailed calculations and simulations may be found in a subsequent paper. This imposes a longitudinal energy oscillation on all of the bunches within the train, causing them to uniformly shift their arrival times and displace the train centroid horizontally proportional to the local dispersion. The typical 
amplitude of this oscillation is relatively large, with the peak fractional energy varying as much as $\pm 7.6 \times 10^{-3}$ for all of the bunches within the train. Due to the RF system's non-linearities, there may be some increase in the energy spread (and bunch length) of the bunches. In the presence of the large energy variation, the transverse field from the stripline kicker deflects the lower energy particles in the bunch (displaced toward the head of the bunch) more than the higher energy particles (displaced toward the tail of the bunch.) Although this is a fairly small differential effect, the bunch is being driven on the head-tail resonance, allowing the head-tail coherent oscillation amplitude to grow.

\subsubsection{Drive-Damp Excitation: Examples}

Two examples of actual drive-damp measurements are found in Figs. 19 and 20. In Fig. 19 the betatron dipole mode amplitude ramps up for the first $3 \mathrm{~ms}$ and then decays exponentially thereafter. Fig. 20 shows one of the head-tail modes being excited. The initial $7 \mathrm{~dB}$ drop in the amplitude of the signal represents the off-resonance excitation of the dipole mode, which immediately switches to oscillating at its resonant frequency (outside of the bandwidth of the receiver) when the drive turns off; the roughly exponential shape thereafter is the head-tail mode decay. As a test, the longitudinal drive to the phase of the RF cavity was turned off, and the head-tail mode exponentially damped signal was observed to vanish into the noise floor.

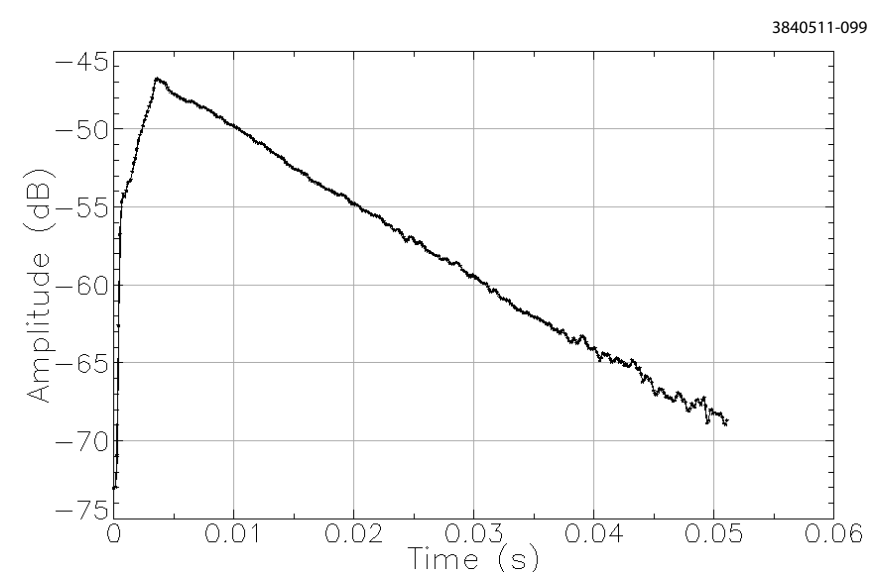

Figure 19. Drive-damp measurement: The trace is the response for the bunch being driven at the vertical betatron frequency. The vertical and horizontal scales are $5 \mathrm{~dB}$ and $10 \mathrm{~ms}$ per division, respectively.

This type of measurement may be very useful for understanding the behavior of bunches within the train before their motion becomes unstable. However, even though much of the data acquisition is automated, there are a few steps in the present version of the data acquisition software, which must be completed manually. In particular the fine adjustment of the spectrum analyzer frequency (centering it on the coherent mode frequency) is necessary to optimize the exponential damping curve. The manual adjustment of the frequency makes this type of measurement fairly timeconsuming. Routinely, after data are taken for several bunches, the beam is topped off. Beam size measurements are typically taken immediately after topping off. 


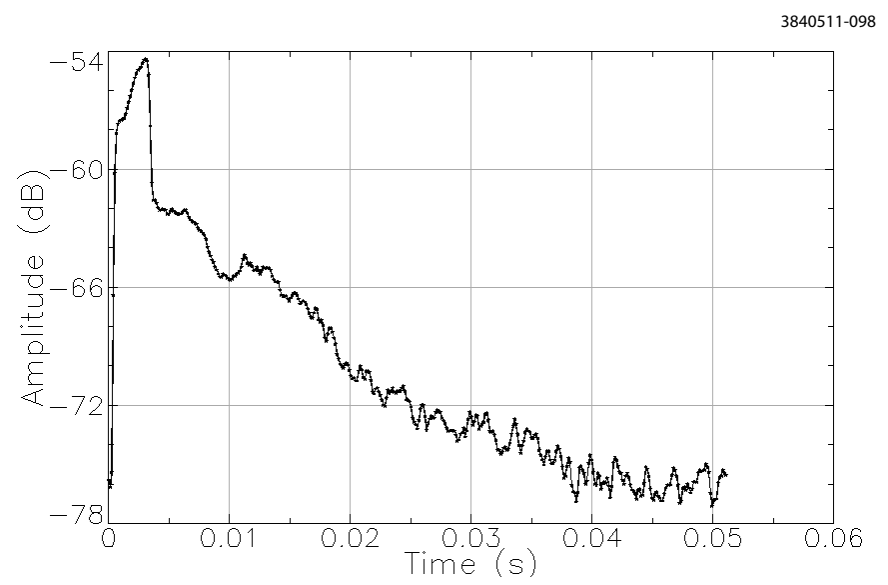

Figure 20. Drive-damp measurement: The trace is the response when one of the head-tail modes is excited. The vertical and horizontal scales are $6 \mathrm{~dB}$ and $10 \mathrm{~ms}$ per division, respectively.

\section{Summary}

This paper has described the instrumentation, which has been developed or modified for use in the CesrTA program for the investigation of storage ring beam dynamics. It also has provided representative examples of actual data and explanations how the basic analysis of some of the data is accomplished. In particular these studies have focused on the methods for low emittance tuning of the beam, on the causes for intra-beam scattering of single bunches and for production and interaction of bunches within trains with electron clouds, produced by photo-electrons from synchrotron radiation and secondary emission.

\section{References}

[1] M. Billing, The conversion of CESR to operate as the Test Accelerator, CesrTA. Part 1: overview, J. Instrum. 10 (July, 2015) .

[2] M. G. Billing and Y. Li, The conversion of CESR to operate as the test accelerator, CesrTA, part 2: Vacuum modifications, J. Instrum. 10 (July, 2015) .

[3] M. G. Billing, J. V. Conway, J. A. Crittenden, S. Greenwald, Y. Li, R. E. Meller et al., The conversion of CESR to operate as the test accelerator, CesrTA, part 3: Electron cloud diagnostics, J. Instrum. 11 (Apr., 2016) .

[4] M. Billing, J. Dobbins, M. Forster, D. Kreinick, R. Meller, M. Rendina et al., Instrumentation for the study of low emittance tuning and beam dynamics at cesr, J. Instrum. 12 (Nov., 2017) .

[5] The CESR Test Accelerator electron cloud research program: Phase i report, Tech. Rep. CLNS-12-2084, LEPP, Cornell University, Ithaca, NY, Jan., 2013.

[6] J. Shanks, Low-Emittance Tuning at CesrTA. PhD thesis, Cornell University, Ithaca, New York, Aug., 2013.

[7] J. Shanks, D. L. Rubin and D. Sagan, Low-emittance tuning at the Cornell Electron Storage Ring Test Accelerator, Phys. Rev. ST Accel. Beams 17 (Apr., 2014). 
[8] M. Ehrlichman, Normal Mode Analysis of Single Bunch, Charge Density Dependent Behavior in Electron/Positron Beams. PhD thesis, Cornell University, Ithaca, New York, May, 2013.

[9] M. P. Ehrlichman, W. Hartung, B. Heltsley, D. P. Peterson, N. Rider, D. Rubin et al., Intrabeam scattering studies at the Cornell Electron Storage Ring Test Accelerator, Phys. Rev. ST Accel. Beams 16 (Oct., 2013) .

[10] K. J. Blaser, A. Chatterjee, M. P. Ehrlichman, W. Hartung, B. Heltsley, D. P. Peterson et al., Measurement of beam size in intrabeam scattering dominated beams at various energies at CesrTA, in IPAC2014: Proceedings of the 5th International Particle Accelerator Conference, Dresden, Germany (C. Petit-Jean-Genaz, G. Arduini, P. Michel and V. R. W. Schaa, eds.), (Geneva, Switzerland), pp. 1635-1637, JACoW, 2014.

[11] J. R. Calvey, Studies of Electron Cloud Growth and Mitigation at Cesr-TA. PhD thesis, Cornell University, Ithaca, New York, Aug., 2013.

[12] J. R. Calvey, G. Dugan, W. Hartung, J. A. Livezey, J. Makita and M. A. Palmer, Measurement and modeling of electron cloud in a field free environment using retarding field analyzers, Phys. Rev. ST Accel. Beams 17 (June, 2014) .

[13] J. R. Calvey, W. Hartung, Y. Li, J. A. Livezey, J. Makita, M. A. Palmer et al., Comparison of electron cloud mitigating coatings using retarding field analyzers, Nucl. Instrum. Methods Phys. Res. A760 (Oct., 2014) 86-97.

[14] J. R. Calvey, W. Hartung, Y. Li, J. A. Livezey, J. Makita, M. A. Palmer et al., Measurements of electron cloud growth and mitigation in dipole, quadrupole, and wiggler magnets, Nucl. Instrum. Methods Phys. Res. A770 (Jan., 2015) 141-154.

[15] J. R. Calvey, W. H. Hartung, J. Makita and M. Venturini, Beam induced electron cloud resonances in dipole magnetic fields, Phys. Rev. Accel. Beams 19 (July, 2016) .

[16] M. Billing, W. Bergan, M. Forster, R. Meller, M. Rendina, N. Rider et al., Beam position monitoring system at cesr, Journal of Instrumentation 12 (2017) T09005.

[17] J. P. Alexander, C. Conolly, E. Edwards, J. W. Flanagan, E. Fontes, B. K. Heltsley et al., Operation of the CESR-TA vertical beam size monitor at eb $=4 \mathrm{GeV}$, Nucl. Instrum. Methods Phys. Res. $\mathbf{A 7 9 8}$ (Oct., 2015) 127-134.

[18] M. P. Ehrlichman, A. Chatterjee, W. Hartung, B. Heltsley, D. P. Peterson, N. Rider et al., Measurement and compensation of horizontal crabbing at the Cornell Electron Storage Ring Test Accelerator, Phys. Rev. ST Accel. Beams 17 (Apr., 2014) .

[19] M. Ehrlichman, A. Chatterjee, W. Hartung, D. P. Peterson, N. Rider, D. Rubin et al., Recent results from CesrTA intrabeam scattering investigations, in IPAC2013: Proceedings of the 4th International Particle Accelerator Conference, Shanghai, China (Z. Dai, C. Petit-Jean-Genaz, V. R. W. Schaa and C. Zhang, eds.), pp. 1126-1128, JACoW, 2013.

[20] M. Ehrlichman, A. Chatterjee, W. Hartung, D. P. Peterson, N. Rider, D. Rubin et al., Experimental study of horizontal-longitudinal coupling at CesrTA, in IPAC2013: Proceedings of the 4th International Particle Accelerator Conference, Shanghai, China (Z. Dai, C. Petit-Jean-Genaz, V. R. W. Schaa and C. Zhang, eds.), pp. 1715-1717, JACoW, 2013.

[21] M. P. Ehrlichman, W. Hartung, M. A. Palmer, D. P. Peterson, N. Rider, D. Rubin et al., Intrabeam scattering studies at CesrTA, in Proceedings of the 2012 International Particle Accelerator Conference, New Orleans, LA, pp. 2970-2972, IEEE, 2012. 
[22] M. G. Billing, G. Dugan, M. J. Forster, R. E. Meller, M. A. Palmer, G. A. Ramirez et al., Measurement techniques to characterize instabilities caused by electron clouds, in Proceedings of the 2011 Particle Accelerator Conference, New York, NY, pp. 1852-1854, IEEE, 2011.

[23] G. Dugan, M. G. Billing, R. Meller, M. Palmer, G. A. Ramirez, J. Sikora et al., Studies of electron-cloud-induced beam dynamics at Cesr-TA, in Proceedings of ECLOUD 2010: 49th ICFA Advanced Beam Dynamics Workshop on Electron Cloud Physics, Ithaca, NY (K. Smolenski, ed.), (Ithaca, NY), pp. 60-75, Cornell University, 2013.

[24] N. Rider, J. Alexander, M. Billing, C. Conolly, N. Eggert, E. Fontes et al., Bunch-by-bunch instrumentation upgrades for CESR, based on requirements for the CESR Test Accelerator research program, in Proceedings of ECLOUD 2010: 49th ICFA Advanced Beam Dynamics Workshop on Electron Cloud Physics, Ithaca, NY (K. Smolenski, ed.), (Ithaca, NY), pp. 88-90, Cornell University, 2013.

[25] M. G. Billing, G. Dugan, M. J. Forster, D. L. Kreinick, R. E. Meller, M. A. Palmer et al., Status of electron cloud dynamics measurements at CESRTA, in Proceedings of the 2011 International Particle Accelerator Conference, San Sebastián, Spain, pp. 799-801, EPS-AG, 2011.

[26] R. L. Holtzapple, R. Campbell, E. L. Holtzapple, M. G. Billing, K. Butler, G. Dugan et al., Dependence of vertical beam dynamics influenced by electron clouds at CesrTA on variations in bunch spacing and vertical chromaticity, in IPAC2013: Proceedings of the 4th International Particle Accelerator Conference, Shanghai, China (Z. Dai, C. Petit-Jean-Genaz, V. R. W. Schaa and C. Zhang, eds.), pp. 1847-1849, JACoW, 2013.

[27] M. G. Billing, G. Dugan, M. Forster, D. L. Kreinick, R. E. Meller, M. A. Palmer et al., Dependence of beam instabilities caused by electron clouds at CesrTA due to variations in chromaticity, bunch current and train length, in Proceedings of the 2012 International Particle Accelerator Conference, New Orleans, LA, pp. 3135-3137, IEEE, 2012.

[28] M. G. Billing, K. R. Butler, G. Dugan, M. J. Forster, R. E. Meller, G. A. Ramirez et al., Observation at CesrTA of the reduction of the vertical beam size of the lead bunch in a train due to the presence of a precursor bunch, in IPAC2013: Proceedings of the 4th International Particle Accelerator Conference, Shanghai, China (Z. Dai, C. Petit-Jean-Genaz, V. R. W. Schaa and C. Zhang, eds.), pp. 1841-1843, JACoW, 2013.

[29] M. G. Billing, K. R. Butler, G. Dugan, M. J. Forster, R. E. Meller, G. A. Ramirez et al., Dependence of beam instabilities caused by electron clouds at CesrTA on variations in bunch spacing and chromaticity, in IPAC2013: Proceedings of the 4th International Particle Accelerator Conference, Shanghai, China (Z. Dai, C. Petit-Jean-Genaz, V. R. W. Schaa and C. Zhang, eds.), pp. 1844-1846, JACoW, 2013.

[30] G. Dugan, M. Billing, K. Butler, J. Crittenden, M. Forster, D. Kreinick et al., Studies at CesrTA of electron-cloud-induced beam dynamics for future damping rings, in Proceedings of the 2012 International Particle Accelerator Conference, New Orleans, LA, pp. 2081-2085, IEEE, 2012.

[31] D. Sagan, R. Meller, R. Littauer and D. Rubin, Betatron phase and coupling measurements at the Cornell Electron/positron Storage Ring, Phys. Rev. ST Accel. Beams 3 (Sept., 2000) .

[32] D. Sagan, Betatron phase and coupling correction at the Cornell Electron/positron Storage Ring, Phys. Rev. ST Accel. Beams 3 (Oct., 2000) .

[33] R. W. Helms and G. H. Hoffstaetter, Orbit and optics improvement by evaluating the nonlinear beam position monitor response in the Cornell Electron Storage Ring, Phys. Rev. ST Accel. Beams 8 (June, 2005). 
[34] D. Sagan and D. Rubin, Linear analysis of coupled lattices, Phys. Rev. ST Accel. Beams 2 (July, 1999) .

[35] M. D. Woodley, J. Nelson, M. Ross, J. Turner, A. Wolski and K. Kubo, Beam based alignment at the KEK-ATF damping ring, in Proceedings of the 2004 European Particle Accelerator Conference, Lucerne, Switzerland, pp. 36-38, EPS-AG, 2004.

[36] D. L. Rubin, M. Billing, R. Meller, M. Palmer, M. Rendina, N. Rider et al., Beam based measurement of beam position monitor electrode gains, Phys. Rev. ST Accel. Beams 13 (Sept., 2010) .

[37] D. Teytelman, iGp-1281F signal processor: Technical user manual, revision 1.7, tech. rep., Dimtel, Inc., San Jose, CA, May, 2009.

[38] J. W. Flanagan, M. Arinaga, H. Fukuma, H. Ikeda, T. Mitsuhashi, J. S. Alexander et al., Single-shot resolution of x-ray monitor using coded aperture imaging, in Proceedings of DIPAC 2011: 10th European Workshop on Beam Diagnostics and Instrumentation for Particle Accelerators, Hamburg, Germany (M. Marx, J. Chrin, I. Martin, R. Mueller and V. R. W. Schaa, eds.), pp. 561-563, 2011.

[39] J. P. Alexander, A. Chatterjee, C. Conolly, E. Edwards, M. P. Ehrlichman, J. W. Flanagan et al., Design and performance of coded aperture optical elements for the CESR-TA x-ray beam size monitor, Nucl. Instrum. Methods Phys. Res. A767 (Dec., 2014) 467-474. 